NIST GCR 16-009

\title{
An Analysis of References from US Patents to NIST-Supported Technical Outputs
}

\author{
Anthony Breitzman Sr, Ph.D. \\ Patrick Thomas, Ph.D. \\ 1790 Analytics, LLC
}

This publication is available free of charge from:

http://dx.doi.org/10.6028/NIST.GCR.16-009

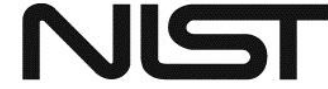

National Institute of Standards and Technology

U.S. Department of Commerce 


\title{
An Analysis of References from US Patents to NIST-Supported Technical Outputs
}

\author{
Prepared for \\ U.S. Department of Commerce \\ Economic Analysis Office \\ National Institute of Standards and \\ Technology Gaithersburg, MD 20899
}

By

Anthony Breitzman, Sr. Ph.D.

Patrick Thomas, Ph.D. 1790 Analytics, LLC

This publication is available free of charge from:

http://dx.doi.org/10.6028/NIST.GCR.16-009

September 2016

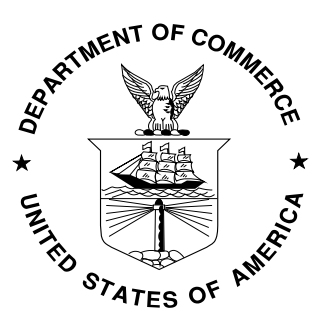

U.S. Department of Commerce

Penny Pritzker, Secretary 


\section{Disclaimer}

This publication was produced as part of contract SB1341-14-SE-0422with the National Institute of Standards and Technology. The contents of this publication do not necessarily reflect the views or policies of the National Institute of Standards and Technology or the US Government. 


\section{Table of Contents}

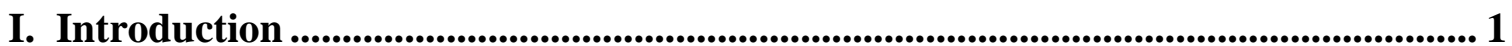

II. Project Design ............................................................................................................................... 1

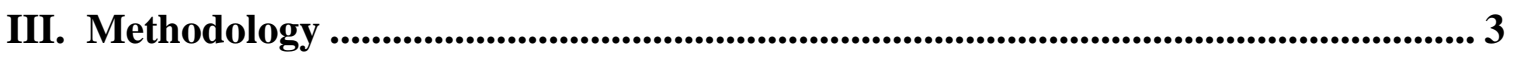

Citing Document Set: Granted US Patents ........................................................... 3

Cited Document Set: NIST Technical Outputs ....................................................... 4

Cited Document Category 1: Patents Assigned to NIST .......................................... 4

Cited Document Category 2: Patents with a NIST Government Interest ................... 4

Cited Document Category 3: NIST-Funded Peer Reviewed Papers .......................... 5

Cited Document Categories 4-11: NIST Grey Literature........................................ 7

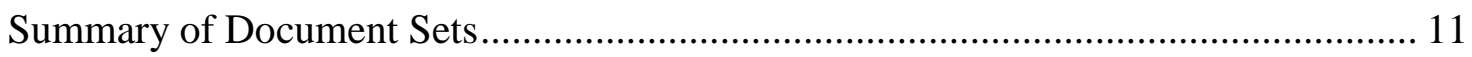

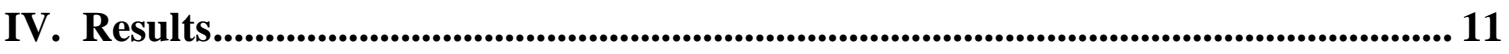

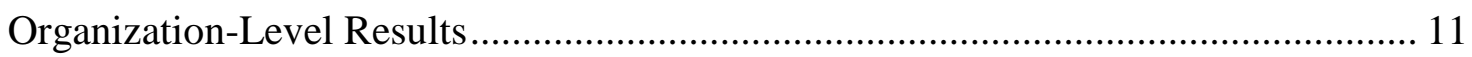

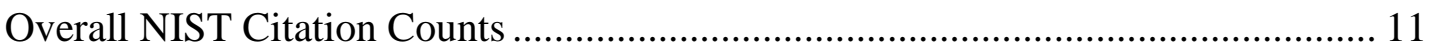

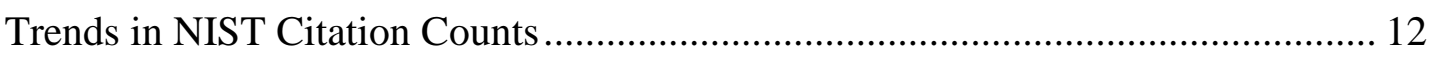

Benchmarking NIST Citation Counts............................................................... 13

Patents Citing NIST Technical Outputs .......................................................... 17

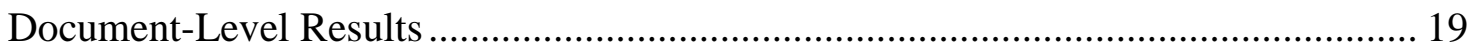

V. Conclusions ............................................................................................................. 22

VI. Appendix: Figures and Tables ..................................................................................... 24 


\section{Executive Summary}

This report outlines the results of an analysis - carried out by 1790 Analytics on behalf of NIST's Economic Analysis Office - designed to evaluate the technological impact of technical outputs associated with NIST.

The analysis is based primarily on locating technical outputs - such as patents, scientific journal articles, standards documents, conferences papers, and other forms of grey literature associated with NIST funding. We then trace forward from these technical outputs to determine how they have influenced subsequent technological developments, specifically those developments described in patents assigned to all organizations.

The idea behind this analysis is that patents that reference NIST technical outputs as prior art build in some way on these NIST outputs. By determining how frequently NIST technical outputs have been cited by subsequent patents, it is thus possible to evaluate the extent to which NIST funded activities have helped form the foundation for a wide range of technologies.

The main findings of this report are:

- Between January 1995 and July 2015, there were a total of 34,241 citations from US patents to NIST-supported technical outputs. This is an impressive figure, especially given the fact that NIST had only 201 US patents assigned to it through the end of 2014. It suggests that NIST's influence extends far beyond just its own patented inventions, and that the agency's activities help form the foundation for technological developments made by a wide range of other organizations.

- We divided the citations from US patents to NIST into four broad categories, based on the type of NIST technical output cited. The four categories are: patents assigned to NIST; patents in which NIST has a government interest; peer reviewed papers funded by NIST; and NIST grey literature.

- $14,538(43 \%)$ of the 34,241 citations from US patents to NIST outputs are to peer reviewed papers authored by NIST-supported researchers; 9,062 (26\%) are to patents in which NIST has a government interest (i.e. patents resulting from research funded by NIST in an external organization); and 2,173 (6\%) are to patents assigned to NIST.

- The remaining $24 \%$ of citations from US patents to NIST technical outputs are to NIST grey literature. The largest of these grey literature categories is software, standard reference databases and algorithms, which accounts for 2,914 (8\%) of citations from US patents to NIST documents. Other grey literature categories that receive significant 
numbers of citations are Official NIST Publications (1,671 citations, or 5\%); Educational Networking (1,267 citations, 4\%); and Other NIST Publications (1,740 citations, $5 \%$ ). The final four grey literature categories together account for only $2.5 \%$ of citations from US patents to NIST documents.

- In terms of trends over time, there was a more than a ten-fold increase in citations from US patents to NIST technical outputs between 1995 and 2014.

- Citations to each of the NIST document types (patents assigned to NIST; patents in which NIST has a government interest; peer reviewed papers funded by NIST; and NIST grey literature) all increased significantly between 1995 and 2014. The largest increases were associated with NIST peer reviewed papers and NIST government interest patents.

- Over the same time period, the overall number of citations provided by US patents also increased significantly. This was due to increases in the number of patents issued each year, and also in the mean number of prior art references listed on patents. We therefore benchmarked citations to NIST against citations to comparable document sets in order to account for these broader changes in the citation landscape.

- We benchmarked citations to NIST patents (both those assigned to NIST, and those in which NIST has a government interest) using our quantitative, normalized patent metrics. These metrics reveal that NIST-supported patents (especially those with a NIST government interest) have a stronger impact, are more generally applicable, and are more original than peer patents of the same age and technology.

- The highest impact NIST-assigned patent (in terms of our normalized Citation Index metric) is US \#5,356,756, which was issued in 1994. This patent describes materials processing for thin films, designed for use in sensors, electronic devices and sample analysis. It has been cited as prior art by 110 subsequent patents, more than seven times as many citations as expected for a patent of its age and technology.

- The highest impact patent in which NIST has a government interest is US \#7,330,404. This patent has been cited by 127 subsequent patents since it was issued in 2008, which is more than 40 times as many citations as expected given its age and technology. It describes a transducer for use in optical storage (for example in disk drives), and is assigned to Seagate Technology.

- We benchmarked citations to NIST peer reviewed papers against citations from the most prolific patenting companies to papers in journals published by the leading 
publishers. Between 2000 and 2014, citations to NIST papers from all patents increased at a faster rate than citations from the patents of leading companies to papers in leading journals. This is an impressive finding, given that the comparison set is highly selective, and only includes leading companies and journal publishers.

- The most highly cited NIST peer reviewed paper was published in Science in 1995, and describes sequencing of the H.influenzae genome (the first example of a free-living organism having its entire genome sequenced). It has been cited by 277 subsequent patents (and over 5,000 papers, according to Google Scholar).

- We benchmarked citations from US patents to NIST grey literature against citations from US patents to grey literature from Massachusetts Institute of Technology (MIT). Overall, citations to NIST grey literature grew almost nine-fold between 2000 and 2014. This is higher than the growth rate associated with citations to MIT grey literature, although the difference is only slight. Even so, outperforming a prestigious institution such as MIT on this metric is an impressive feat.

- The most highly cited items of NIST grey literature are the Federal Information Processing Standards (FIPS) publications outlining the Advanced Encryption Standard (FIPS 197); the Secure Hash Standard (FIPS 180); and the Digital Signature Standard (FIPS 186). These standards documents have been cited by hundreds of subsequent US patents.

- We also analyzed the US patents that cite NIST technical outputs as prior art, focusing on the distribution of these patents by technology, industry, and assignee.

- More than one-fifth of the $34,000+$ citations to NIST come from patents related to Computer Hardware \& Software; and more than one-third are from that category plus Communications. Other major citing categories are Measuring \& Testing, and Semiconductors \& Electronics.

- A large number of citations from Computer Hardware \& Software and Communications patents are to FIPS documents such as the Secure Hash Standard (FIPS 180) and the Advanced Encryption Standard (FIPS 197). Also highly cited are various NISTIRs and NIST special publications, which often describe the contents of the FIPS documents.

- IBM and Microsoft cite NIST most extensively, followed by Calcitec (a medical devices firm), 3M and Micron Technology. Both IBM and Microsoft build heavily on NIST grey literature, as do Intel, Cisco and Hewlett-Packard. Meanwhile, Calcitec 
builds primarily on NIST peer reviewed papers, as do the University of California and Michigan State University, plus the US Department of Commerce (which includes citations from NIST's own patents to NIST papers). NIST's patents are cited most heavily by $3 \mathrm{M}$, Western Digital and Cree.

- In terms of industries, Universities cite NIST technical outputs most extensively, followed by Semiconductor companies, Software companies, and Communications companies. Other industries that cite NIST outputs extensively are Medical Equipment/Instruments, Computer Systems and Electronics.

- Overall, the analysis presented in this report reveals that there has been a significant increase over time in citations from US patents to NIST technical outputs. These citations are from patents owned by organizations across a broad range of industries, describing a wide variety of technologies. Our analysis also demonstrates how the increase in citations to NIST outstrips citations to comparable document sets. This suggests that NIST's technical outputs have had a strong, and growing, impact on technological developments. 


\section{Introduction}

This report outlines the results of an analysis designed to evaluate the technological impact of technical outputs associated with the National Institute of Standards and Technology (NIST). NIST funds a wide range of technical activities and research, resulting in many different types of published outputs. However, NIST itself has relatively few patents assigned to it. In order to assess NIST's influence on technological developments, it is thus necessary to look beyond simply the agency's portfolio of granted patents.

The analysis presented in this report is designed to provide a detailed evaluation of NIST's technological impact. It is based primarily on locating technical outputs - such as patents, scientific journal articles, standards documents, conferences papers, and other forms of grey literature - associated with NIST funding. We then trace forward from these technical outputs to determine how they have influenced subsequent technological developments, specifically those developments described in patents assigned to all organizations. In this way, it is possible to discern NIST's impact on innovations developed by organizations of all types and sizes, across all technologies and industries.

This report contains three further sections. The first of these sections provides an overview of the project design, and the evaluation techniques used in the analysis. The second section outlines the methodology used to carry out the analysis, including how the various data sets were constructed. The third section contains the results of the analysis, examining the impact of NIST funded technical outputs on subsequent technological developments.

\section{Project Design}

The analysis presented in this report centers upon connections between generations of research that are made by prior art references listed on patent documents. The purpose of these prior art references is to detail the state of the art at the time of the patent application, so that the applicant can demonstrate how the new invention is original over and above this prior art. These references may consist of many different types of documents. A large number of the references are to earlier patents and published patent applications; while others are to scientific journal articles and conference proceedings; and others are to standards, technical reports, magazines and newspapers.

The responsibility for adding prior art references differs across patent systems. In the US system, it is the duty of patent applicants and their attorneys to reference (or 'cite') all prior art of which they are aware that may affect the patentability of their invention. Patent 
examiners may then reference additional prior art that limits the claims of the patent for which an application is being filed.

Patent citation analysis centers upon the links between generations of research that are made by these prior art references. In basic terms, this type of analysis is based upon the idea that the prior art referenced in patents has had some influence, however slight, upon the development of these patents. The prior art is thus regarded as part of the technical foundation for the later inventions.

In assessing the influence of individual documents - whether patents, papers or other forms of grey literature - citation analysis centers on the idea that highly cited documents (i.e. those cited by many later patents) tend to contain technological information of particular interest or importance. As such, they form the basis for many new innovations and research efforts, and so are cited frequently by later patents. While it is not true to say that every highly cited document is important, or that every infrequently cited document is necessarily trivial, many research studies have shown a correlation between patent citations and measures of technological and scientific importance. For background on the use of patent citation analysis, including a review of validation studies supporting its use, see Breitzman A. \& Mogee M. "The many applications of patent analysis", Journal of Information Science, 28(3), 2002, 187-205. For a detailed study of various hypotheses surrounding the motivations of inventors in citing non-patent references, see Branstetter, L. and Oguara, Y. "Is Academic Science Driving a Surge in Industrial Innovation? Evidence from Patent Citations", NBER Working Paper 11561, August 2005.

Patent citation analysis has also been used extensively to trace technological developments. For example, in the analysis presented in this report, we examine citations from patents to earlier technical outputs resulting from NIST funded activities. The idea behind this analysis is that the patents have built in some way on these NIST technical outputs. By determining how frequently NIST funded technical outputs have been cited by subsequent patents, it is thus possible to evaluate the extent to which NIST funded activities have helped form the foundation for a wide range of technologies.

It should be noted that the analysis presented in this report is based on prior art references from US patents to NIST funded technical outputs, such as patents, journal articles, conference papers, standards and software. The analysis does not consider references from journal papers to NIST funded papers (or from journal papers to NIST funded patents, although references from papers to earlier patents are very rare). This is purposeful, since our primary focus is on how NIST activities have influenced innovations developed across a range of industries. Such innovations are particularly likely to be protected by patents. Meanwhile, scientific papers may describe more early-stage and exploratory research, so 
references from them to NIST papers may be mainly a reflection of the scientific, rather than technological, impact of the agency's activities.

\section{Methodology}

While the previous section outlines the general idea behind the analysis described in this report - i.e. tracing citation links from US patents to earlier NIST funded technical outputs - this section describes the methodology used to implement the analysis. In particular, it focuses on how these two document sets - US patents and NIST funded technical outputs - were collated, and how citation links between them were located.

\section{Citing Document Set: Granted US Patents}

In order to perform citation linkage analyses such as the one described in this report, 1790 Analytics maintains our own internal patent databases. Currently, we have every granted US patent and published US patent application from 1969 to date in our US patent database (which was used in this project). We also have every patent from 1978 to date in our EPO (European Patent Office) database; and every PCT (Patent Cooperation Treaty) application from 1978 to date in our WIPO (World Intellectual Property Organization) database.

Our patent databases contain detailed information on all patents, including inventors, assignees, titles, abstracts, patent classifications, and application and issue dates. In addition, our databases contain all prior art references listed on the front page of patents, including references to earlier patents, and to other non-patent literature. It is this detailed prior art reference information that makes possible large-scale citation studies such as the one outlined in this report.

One other important feature of our patent databases is our corporate thesaurus, which accounts for the many different names under which organizations own patents. As an example, the pharmaceutical firm Sanofi has been assembled through the merger and divestiture of companies that were themselves built through acquisition. Sanofi has patents under $200+$ different company names, and fewer than $10 \%$ of its patents are assigned to a name that contains 'Sanofi' within the string. Our corporate thesaurus automatically accounts for these subsidiaries and provides us with a complete patent inventory for each organization. This is an important element of the analysis presented in this report, particularly with regard to the results showing organizations whose patents reference NIST technical outputs most extensively. 


\section{Cited Document Set: NIST Technical Outputs}

There are four main categories of NIST technical outputs included in the analysis described in this report: patents assigned to NIST; patents in which NIST has a government interest; peer reviewed papers funded by NIST; and NIST grey literature (which is further divided into eight sub-categories). Below, we outline the processes involved in compiling each of these sets of technical outputs, categorizing them (in the case of NIST grey literature), and locating prior art references to them from US patents.

\section{Cited Document Category 1: Patents Assigned to NIST}

Collating Documents - NIST provided us with a list of patents assigned to the agency, so this was the most straightforward document set to collate from our perspective.

Locating Prior Art References from US Patents - prior art references to patents are listed in the form of patent numbers. For example, US Patent \#9,012,542 references nine earlier granted US patents as prior art, and these patents are identified by their patent numbers $(\# 2,388,169$; \#3,450,667 etc). This patent also references five published US patent applications, and three foreign patents, all of which are again listed by their patent numbers.

Patent numbers (for both granted patents and published patent applications) are unique identifiers, and are typically listed in a standard format. As a result, it is possible to construct databases containing all citation links between generations of patents, such as the database we maintain at 1790 . The citation links consist of pairs of citing-cited patent numbers, with both of these numbers being unique identifiers. Given access to such a database, it is relatively straightforward to take a starting set of patent numbers (i.e. the cited half of the pair), and locate subsequent patents that reference these patents as prior art (i.e. the citing half of the pair). In this analysis, we carried out such a process, with the starting set consisting of the patent numbers associated with the patents assigned to NIST.

\section{Cited Document Category 2: Patents with a NIST Government Interest}

Collating Documents - US patents have on their front page a section entitled 'Government Interest'. This section details the rights that the government has in a particular invention. These typically exist where a government agency funds research at an external organization (such as a for-profit company or a non-profit organization), and the government has certain rights to the patents resulting from this research. For example, US Patent \#9,024,298, which is assigned to Xerox Corporation, contains the following statement in its Government Interest field: 
This invention was made with United States Government support under Cooperative Agreement No. 70NANBOH3033 awarded by the National Institute of Standards and Technology (NIST). The United States Government has certain rights in the invention.

We searched the Government Interest field of all US patents, and identified those patents that refer to NIST. These searches included various forms of the full agency name National Institute of Standards and Technology - plus the NIST acronym.

Locating Prior Art References from US Patents - we identified the patent numbers for the NIST government interest patents. We then searched in our databases for subsequent US patents that reference these NIST government interest patents as prior art. As in the case of NIST assigned patents, this was a relatively straightforward process involving matching patent numbers, which are unique identifiers.

\section{Cited Document Category 3: NIST-Funded Peer Reviewed Papers}

Collating Documents - NIST provided us with a list of 50,000+ peer reviewed papers authored by NIST personnel. These papers were downloaded by NIST personnel from the Web of Science ${ }^{1}$.

Locating Prior Art References from US Patents - prior art references to items other than patents are typically referred to as Non-Patent References (NPRs). These NPRs can be to any published document, from scientific journal articles, conference papers and standards documents, all the way to comic strips and brochures. They are much more difficult to work with than prior art references to patents, since they are in free text. Also, inventors are not required to use a standard referencing format for listing NPRs, in contrast to an author submitting a manuscript for publication in a scientific journal. In addition, there is no equivalent to a journal editor or reviewer ensuring that the references are complete, since this is typically not a major concern of the patent examiner. As a result of these various factors, NPRs are thus free text that can be in any format, and may be incomplete. Author names may be present or absent, and journal names can be in many different formats (for example, there are more than 30 variant spellings of the journal title Journal Of Thoracic And Cardiovascular Surgery found in patents, including misspellings of thoracic and different ways of abbreviating cardiovascular).

\footnotetext{
${ }^{1}$ This document set consists primarily of peer reviewed journal articles, but it does also contain some papers from conference proceedings, some of which will be peer reviewed and some not. We define this category as peer reviewed papers to make it distinct from the so-called 'grey literature' categories consisting of white papers and internal NIST publications such as NISTIRs, standards recommendations etc.
} 
We have built a number of tools designed specifically to address these issues with NPRs. We applied these tools here to locate prior art references from patents to peer reviewed papers authored by NIST funded researchers. This process involved matching the NPRs listed on all US patents against the 50,000+ NIST papers downloaded from the Web of Science.

These Web of Science records are in a standard format, listing the year, journal, first author, first page, and title for each NIST funded paper. We thus processed NPRs into the same format, parsing them in order to isolate these various reference elements. We then applied our journal thesaurus to the journal element of each paper. This thesaurus contains more than 50,000 variant names for journals. For example, the Journal of the American Medical Association may appear as J. Am. Med. Assoc., Jrnl Am. Med Assoc., J of Am Med Assoc., $J A M A$, or one of more than a dozen other variants.

At this point, we had two lists - one containing NPRs, and one containing NIST papers each with the various bibliographic elements (year, journal, author, page, title) all extracted and standardized. We then matched these two lists, and scored the quality of each match according to the following scoring system:

\begin{tabular}{cl}
\hline Score & Fields Matched \\
\hline 10 & Year, Journal, Author, Page \\
\hline 9 & Year, Journal, Author6, Page \\
8 & Year, Journal10, Author, Page, Title15 \\
7 & Year, Journal, Author6, Title15 \\
6 & Journal, Author, Title15, Page \\
5 & Journal, Author, Full Title \\
4 & Year, Journal, Author, Volume OR Title \\
\hline
\end{tabular}

The highest scoring match is thus one where the year, journal name, first author and first page number all match perfectly. In the second-highest scoring match, the year, journal name and first page number all match, along with six characters of the first author name (Auth6). For example, a name like Petropoulos may be misspelled in multiple ways, but a match of six characters is likely to mean a correct match. Similarly, in the third-highest scoring match, ten characters of the journal name (Journal10) and fifteen characters of the title (Title15) must match in order to achieve this score.

Under this scoring system, matches with a score of 8-10 are automatically considered to be correct. Matches with a score of 4-7 are considered to be possible correct matches, and were confirmed or discarded via human inspection. 


\section{Cited Document Categories 4-11: NIST Grey Literature}

Collating Document Set - whereas NIST provided us with a list of peer reviewed papers associated with its funding, there was no similar list of NIST grey literature available (and such a list would be extremely difficult and time-consuming to construct). The process for locating NIST grey literature cited by US patents was thus somewhat different from the process used to locate other NIST technical outputs cited by patents. Specifically, the process for other technical outputs involved two steps - i.e. first identifying the universe of NIST outputs, and then matching these outputs to references in US patents. Meanwhile, the process for identifying citations to NIST grey literature only involved a single step i.e. references from US patents to grey literature were located directly, without first defining the universe of this literature.

Locating Prior Art References from US Patents - we identified grey literature cited in US patents by searching the non-patent references in these patents for keywords related to NIST, such as NBS, Nat*Bur*St*, Nat*Inst*St*, FIPS, Fed*Inf*Proc*St*, NIST* etc. We also accounted for possible term confusion, for example searching for NIST* and not machinist* or administration.

Categorizing Cited NIST Grey Literature - NIST personnel expressed significant interest regarding which types of grey literature produced by the agency have been particularly influential upon subsequent technological developments. We thus categorized NIST grey literature into eight subcategories, as defined by NIST personnel. This was a complicated process, and involved a series of iterations using combinations of keywords and manual review in order to categorize NIST grey literature satisfactorily.

The eight categories of grey literature (numbered 4-11, following on from the three categories of NIST technical outputs already discussed), are outlined below. The constituent elements of each category are listed, along with examples of technical outputs associated with the category.

\section{Category 4 - Educational Networking}
a. Conferences
b. Seminars
c. Webinars
d. Workshops
e. Symposiums
f. Presentations
g. Short Courses
h. Proceedings 


\section{Examples:}

Holleyman, Robert W. "On the Export of Software with Encryption Capabilities ", testimony presented at Key Escrow Meeting, NIST, Gaithersburg, MD, Sep. 6, 1995, pp. $1-7$.

Sweat et al. Characterization of natural and induced variation in the LOV1 gene, a CCNBS-LRRR gene conferring victorin sensitivity and disease susceptibility in Arabidopsis. MPMI. 2008. 21(1): 7-19.

Self-Organizing Neural Network Character Recognition on a Massively Parallel Computer, Wilson et al, NIST, International Joint Conference on Neural Networks, Proceeding, II, pp. 325 329, Jun. 1990, San Diego, Calif.

\section{Category 5 - Software/Standard Reference Databases/Algorithms}
a. Materials and Property Data
b. Standard Reference Data
c. Software (ex: NSRL)
d. Databases (excluding SRDs)
e. FIPS
f. NIST WebBook
g. Documentation/Publications Regarding SRMs (that do not have a NIST- assigned publication number)
h. Joint Partnership Publications Regarding Standards (ex: ANSI/NIST Doc category)
i. $\quad$ NIST Standard Reference Data Series (NIST NSRDS)

\section{Examples:}

NIST, “Advanced Encryption Standard (AES)", FIPS Publication 197, 52 pages, Nov 26, 2001.

http://www.nsrl.nist.gov/, National Software Reference Library, printed from website May 15, 2012, 1 page.

Grother, Patrick J. "NIST special database 19 handprinted forms and characters database." National Institute of Standards and Technology (1995).

\section{Category 6 - Standard Reference/Resource Materials/General Information}
a. Measurement and Testing Methodology
b. Standard Reference Materials (SRMs)
c. Website Citation (ex: NIST Home Page)
d. Tables
e. Dictionaries
f. Stand-Alone Pictures
g. Fact Sheets 
Examples:

Periodic Table. NIST. 2003.

Photograph of the NIST Micro Microwave Oven, NISTmicropic.jpg, Mar. 1, 2011.

Semicon West 2001, Website:

http://www.nist.gov/public_affairs/factsheet/semiconwest01.htm.

Category 7 - Official NIST Publications (not peer-reviewed journal)

a. NIST Special Publications (NIST SP-XXX)

b. NIST Technical Notes (NIST TN)

c. $\quad$ NIST Grant/Contract Reports (NIST GCR)

d. NIST Handbook

e. $\quad$ NIST Interagency/Internal Reports (NISTIR)

Examples:

Ross, U.S. Department of Commerce, Nat'1 Bur. Stands, Publication NSRDS-NBS59 (Jan. 1977).

Bearden, J. A.; X-ray Wavelengths and X-ray Atomic Energy Levels. US Dept of Commerce, Nat'l Bur. Stands. NSRDS 14, pp. 1-47 (Sep. 1967).

Burr et al., Ed., "Electronic Authentication Guideline," Version 1.0.2, http://csrc.nist.gov/publications/nistpubs/800-63/SP800-63V1(sub)—0(sub)—2.pdf, Apr. 2006, 64 pages.

\section{Category 8 - Joint Partnership Publications (not peer-reviewed journal)}
a. DARPA
b. NSA
c. Colorado School of Mines
d. JILA
e. JQI
f. HML
g. IBM

Examples:

Federal Aviation Administration (FAA), "Draft Burnthrough Test Standard for Aircraft Insulation," FAA on World Wide Web (http://www.fire.tc.faa.gov/burnthru.html), p. 16, (Mar. 24, 1999).

Technisches Messen, vol. 51, 1984, Issue No. 3, pp. 8395 Federal Physical Technical Institute (Physikalisch Technischen Bundesantalt) or the Nat'l Bur. Stands. 


\section{Category 9 - Other NIST (non peer-reviewed) publications}
a. Circulars
b. Internal Reports
c. Recommendations
d. Proposals
e. Bulletins
f. $\quad$ Press Releases

\section{Examples:}

US Dept of Commerce, NIST, FY 2010 Small Business Innovation Research Program Solicitation, NIST-10-SBIR, Oct. 30, 2009-Jan. 22, 2010.

Proctor, F.; "Validation of Standard Interfaces from a Machine Control", 1996, NIST, NIST Internal Report, pp. 659-664.

Snoke, Hubert R., Building Mat ls \& Structures, Report BMS 70, Nat'l Bur. Stands, 1941, p. 15.

\section{Category 10 - Communication/Correspondence/Inquiries}

a. Questions, answers, and requests for comments on FIPS

b. FFOs, solicitations, private communications

Examples:

Peter L. Bender, Nat'l Bur. Stands, private communication, 1978

Leonard E. Miller, Apr. 2002, NIST.

\section{Category 11 - Other}

a. NIST news clips - when NIST is mentioned in a newspaper, article, etc.

b. Articles reporting NIST technical outputs

Examples:

English translation of Official Letter of Taiwan Patent Office (Nat'l Bur. Stands) in Taiwan Appln. No. 82100814.

Messmer, Ellen, "NIST proposes standard for electronic signatures", Network World, p. 4, Jul. 1991. 


\section{Summary of Document Sets}

As a result of the processes outlined above, we compiled two data sets, the first consisting of granted US patents, and the second containing NIST funded technical outputs referenced as prior art by these patents. These NIST outputs are categorized as follows:

\begin{tabular}{cl}
\hline Category Number & Category Definition \\
\hline 1 & NIST-Assigned Patents \\
2 & NIST Government Interest Patents \\
3 & NIST-Authored Peer-Reviewed Publications \\
4 & Educational Networking \\
5 & Software/Standard Reference Databases/Algorithms \\
6 & Standard Reference/Resource Materials/General Information \\
7 & Official NIST Publications (not peer-reviewed journal) \\
8 & Joint Partnership Publications (not peer-reviewed journal) \\
9 & Other NIST (non peer-reviewed) publications \\
10 & Communication/Correspondence/Inquiries \\
\hline 11 & Other \\
\hline
\end{tabular}

These document sets, and the citation links between them, form the basis for our analysis of the impact of NIST funded research on subsequent technological developments. The results of this analysis are outlined in the following section of the report.

\section{Results}

We report the results of our analysis at two different levels of granularity. First, we report organization-level results, examining the overall impact of NIST funded technical outputs, both in absolute terms, and in the context of the broader citation landscape. We then outline results at the level of individual NIST technical outputs, highlighting those published items that have had a particularly strong impact on subsequent technological developments.

\section{Organization-Level Results}

\section{Overall NIST Citation Counts}

Between January 1995 and July 2015, there were a total of 34,241 citations from US patents to NIST-supported technical outputs. This is an impressive figure, especially given the fact that NIST had only 124 US patents assigned to it through the end of 2014. It suggests that NIST's influence extends far beyond just its own patented inventions, and that the agency's 
activities help form the foundation for technological developments made by a wide range of other organizations.

Figure 1 contains a breakdown of the 34,241 citations from US patents, according to the category of NIST document receiving each citation. This figure reveals that 14,538 (43\%) of the citations are to peer reviewed papers authored by NIST-supported researchers. The next largest category is NIST government interest patents, which received 9,062 (26\%) of the citations. These are patents assigned to organizations other than NIST, but in which NIST has some rights having provided funding for the research that led to the patented inventions. The number of citations to NIST government interest patents is more than four times higher than the number of citations to patents actually assigned to NIST $(2,173$, or $6 \%$ ). This reflects that fact that NIST supports a great deal of research carried out by other organizations, rather than only funding researchers within the agency.

The remaining $24 \%$ of citations from US patents to NIST documents are to various types of grey literature. The largest of these grey literature categories is software, standard reference databases and algorithms, which accounts for 2,914 (8\%) of citations from US patents to NIST documents. Other grey literature categories that receive significant numbers of citations are Official NIST Publications (1,671 citations, or 5\%); Educational Networking (1,267 citations, 4\%); and Other NIST Publications (1,740 citations, 5\%). The final four grey literature categories together account for only $2.5 \%$ of citations from US patents to NIST documents.

Figure 2 is based on the same data as Figure 1, but presents it in the form of a pie-chart. Also, in this figure, all of the grey literature categories are combined for ease of presentation. As such, Figure 2 is a basic overview of citations from US patents to NIST technical outputs in the four main categories: peer reviewed papers; government interest patents; assigned patents; and grey literature.

\section{Trends in NIST Citation Counts}

Figure 3A shows trends over time in citations from US patents to NIST technical outputs. Specifically, this figure shows the number of citations to NIST each year from 1995 to 2014, along with the number of unique US patents citing NIST each year (the two are not equivalent, since one patent may reference multiple NIST documents as prior art). The first point to note in Figure $3 \mathrm{~A}$ is the growth in the number of citations from US patents to NIST. There was a more than a ten-fold increase in these citations between 1995 and 2014. The second point to note is the widening gap between the two lines in this figure, especially in recent years. In the 1990s, patents that cited NIST typically cited a single NIST document, so the number of citations and the number of citing patents were almost identical. More recently, the two numbers have started to diverge, to the point where, in 
2014, there were 3,000 unique patents that cited NIST, and these patents provided more than 4,500 citations to NIST (i.e. an average of more than 1.5 citations per citing patent).

Beyond these overall trends, it is informative to examine whether there is any difference in the citation trends across different types of cited NIST technical outputs. Figure 3B shows the number of citations to the four main types of NIST outputs (peer reviewed papers; government interest patents; assigned patents; and grey literature) from US patents issued each year from 1995 through 2014. This figure reveals that the sharp increase in citations to NIST outputs shown in Figure 3A can also be seen for each of the four document types. Having said this, while citations to each of the NIST document types have increased over time, the largest increases are associated with peer reviewed papers and government interest patents.

The trend lines in Figures 3A and 3B suggest that the influence of NIST documents has increased markedly over the past two decades. However, it should be noted that the overall number of citations provided by US patents has also increased significantly over the same time period. This is due to increases in the number of patents issued each year, and also in the mean number of prior art references listed on patents. Also, over time, the corpus of NIST technical outputs has grown, so there are many more such outputs available to be cited. In subsequent figures, we attempt to account for these various factors, and thus place the growth of citations to NIST technical outputs in the context of the wider citation landscape.

This wider landscape may differ for the various types of cited document. For example, trends over time in citations to patents may be different to trends in citations to journal articles. Also, as shown in Figure 3B, there are differences in the rate of growth in citations to the different types of NIST technical outputs. Given these factors, we examine each type of NIST technical output (i.e. patents, peer reviewed literature, grey literature) separately, as outlined below.

\section{Benchmarking NIST Citation Counts}

To assess NIST patents (both those assigned to the agency and those with a NIST government interest) within the wider citation landscape, we employed our citation-based patent metrics. These metrics are used across many of our projects, and form the basis for the Patent Power Scorecards published annually in IEEE Spectrum magazine (an example of these scorecards is available at http://spectrum.ieee.org/static/interactive-patent-power2015). The metrics - which are described below - assess different characteristics of patents, notably their impact, generality and originality. 
As discussed earlier, one widely used method of assessing the impact of a patent is to count how many times it has been cited by subsequent patents. However, using raw citation counts to assess patent impact is often problematic, since older patents are likely to be more highly cited, simply because they have had more time to accrue citations from later patents. Citation rates also differ across technologies. In order to overcome these two problems, we normalize citation counts by issue year and technology (as defined by Patent Office Classifications) to produce a Citation Index for all granted patents.

The Citation Index is derived by dividing the number of citations received by a patent by the mean number of citations received by all patents from the same Patent Office Classification (POC) and issue year. For example, the number of citations received by a patent issued in 2005 with a primary POC of 351/210 is divided by the mean number of citations received by all patents issued in 2005 with the same primary POC.

The expected Citation Index for an individual patent is one. The extent to which a patent's Citation Index is greater or less than one reveals whether it has been cited more or less frequently than expected, and by how much. For example, a Citation Index of 1.5 shows that a patent has been cited $50 \%$ more frequently than expected. Meanwhile a Citation Index of 0.7 reveals that a patent has been cited $30 \%$ less frequently than expected.

Patent generality is based on the premise that technologies with applications across a range of industries are likely to be of greater interest than technologies with only a narrow range of applications. For example, a new material that is used in many different industries is regarded as more general than a material that is only used in a single application.

This metric is operationalized via citation links. Specifically, patents cited as prior art by subsequent patents from numerous technologies are regarded as having more general applicability than patents that are only cited by subsequent patents from the same technology. We normalize generality by both issue year and technology (as defined by Patent Office Classifications), to derive a Generality Index with an expected value of one. Patents with a Generality Index greater than one are more general than peer patents drawn from the same technology and issue year, while patents with a Generality Index below one are less general than their peers.

Patent Originality is the analog of Patent Generality, and looks backwards in time, rather than forwards. It is based on the premise that patents that build upon a wide range of technologies are likely to be more original than patents that draw only upon earlier developments in the same technology (which may represent incremental improvements on that technology). This indicator is once again operationalized via citation links. 
Specifically, patents that cite as prior art earlier patents from numerous technologies are regarded as more original than patents that only cite patents from the same technology.

We again normalize originality by both issue year and technology (as defined by Patent Office Classifications) to derive an Originality Index with an expected value of one. Patents with an Originality Index greater than one are more original than peer patents drawn from the same technology and issue year, while patents with an Originality Index below one are less original than their peers.

Figure 4 shows the Citation Index, Generality Index and Originality Index for patents assigned to NIST and for patents in which NIST has a government interest (i.e. those patents resulting from research that NIST funded in external organizations). The metrics are shown for two separate time periods - i.e. NIST-related patents issued in 2005-09 and NIST-related patents issued in 2010-2014.

The Citation Index for NIST-assigned patents is below one for both time periods, suggesting that these patents have not had a particularly strong impact on subsequent technological developments. However, the number of patents assigned to NIST is very small (only 22 patents were granted to NIST in 2005-09 and 100 in 2010-2014), so not too much importance should be attached to this finding.

The Citation Index for NIST government interest patents is much more impressive, exceeding 1.6 in both time periods. This shows that the NIST government interest patents have been cited more than $60 \%$ more frequently than expected, given their relative age and technology. In turn, this suggests that they have had a strong impact on subsequent innovations. Given that the number of NIST government interest patents is much higher than the number of NIST-assigned patents, this finding is much more noteworthy.

A similar pattern can be seen for NIST-assigned patents and NIST government interest patents in terms of their Generality Index. Again, NIST-assigned patents perform relatively poorly on this metric, with a Generality Index below one in both time periods, albeit based on a small number of patents. Meanwhile the Generality Index for NIST government interest patents is much higher, and exceeds two in the most recent time period. This suggests that the NIST government interest patents have a broad applicability across a range of technologies.

The results for the two sets of patents are much more similar in terms of their Originality Index. In both cases, the Originality Index exceeds one for both time periods, but not by a wide margin. This suggests that both the NIST-assigned patents and the NIST government interest patents are more original than expected, but not by a significant degree. 
Overall, the patent citation metrics in Figure 4 support the idea that NIST-supported patents, especially those with a NIST government interest, have had a strong, wide-ranging impact on subsequent technology developments. As such, the increases in citations to NIST patents reported in Figure 3B appear to be associated with this strong impact, rather than simply being a reflection of changes in the underlying citation landscape.

Placing citations to NIST's peer reviewed papers and grey literature in a wider context is more difficult than it was for citations to NIST-supported patents. There are no equivalent normalizations measuring how many times a paper of a certain age, and from a given subject area, can be expected to have been cited in subsequent patents. Given this lack of available normalizations, we had to devise suitable comparisons in order to place the citations to NIST literature in a wider context.

In evaluating citations to NIST published documents, we examined peer reviewed papers and grey literature separately. For peer reviewed papers, one obvious approach would have been to compare citations to NIST's papers against citations to papers produced by other agencies or institutions. However, this would have required identifying complete paper sets for those institutions, and then doing a match similar to that described in the methodology section above. That is a costly endeavor, and is well beyond the scope of this project.

In recognition of the time and budget constraints, our comparison set for NIST peer reviewed papers was instead drawn from one of our existing projects. Each year, we produce a report for the Institute of Electrical and Electronics Engineers (IEEE). In this project, we identify the 40 companies with the largest number of US patents granted in the most recent year. These companies include IBM, Siemens, Canon etc. We then locate and disambiguate prior art references from the patents of these companies to papers published in journals from the leading publishers, specifically Elsevier, Wiley, Springer, American Chemical Society, American Institute of Physics, IEEE, IOP Publishing, and Optical Society of America.

In the current analysis, we used the annual citation counts from this IEEE project as a benchmark against which to compare citation counts to NIST peer reviewed papers. It is worth noting that more than $65 \%$ of NIST's peer reviewed papers are published in a journal from one of these leading publishers, so this seems like a reasonable comparison set.

Figure 5A contains an index (base year $=2000$ ), showing the growth in the number of citations from the IEEE analysis, plus the growth over the same period in the number of citations from all US patents to NIST peer reviewed papers. This figure reveals that citations from the leading companies to the leading publishers increased more than six-fold between 2000 and 2014. While impressive, this increase is still lower than that associated 
with NIST papers. Between 2000 and 2014, the number of citations from US patents to NIST papers increased seven-fold.

The IEEE analysis is based on a highly select set of citing and cited documents - i.e. citations from patents owned by leading companies to papers published in leading academic journals. Hence, the fact that citations to NIST papers have grown faster than citations to papers in the leading journals is highly impressive. If we were to add journals from other less renowned publishers, we suspect that the outperformance of NIST papers would be even more marked (however, we do not have data on citations to these journals readily available).

With respect to NIST grey literature, finding a reasonable benchmark was also problematic. NIST produces a wide variety of types of grey literature, from standards documents, to official definitions, to software, to reference materials. There are no comparable organizations that produce as wide a variety of grey literature as NIST. The best comparison we could locate was Massachusetts Institute of Technology (MIT). Like NIST, MIT produces a broad range of technical reports, thesis drafts, software, and guides. Moreover, it is one of the most respected and entrepreneurial universities, with many of its students and faculty creating hi-tech startup companies. As such, MIT's technical outputs are likely to be closely related to the applied technologies described in patents.

MIT should thus be a suitable (and indeed demanding) comparison for NIST with regard to grey literature. Figure 5B shows an index (base year $=2000$ ) for citations each year from all US patents to grey literature produced by NIST and MIT. Overall, citations to NIST grey literature grew almost nine-fold between 2000 and 2014. This is higher than the growth rate associated with citations to MIT grey literature, although the difference is only slight. Even so, outperforming a prestigious institution such as MIT on this metric is an impressive feat.

\section{Patents Citing NIST Technical Outputs}

Having examined overall trends in citations to NIST, and their distribution across different NIST document types, we now look in more detail at the patents that cite NIST technical outputs as prior art. We focus in particular on the distribution of these patents by technology, industry, and assignee.

Figure 6 shows citations to the four main types of NIST documents by the technology area of the citing patents. This technology area is defined based on 1790's technology concordance, which maps patents to technologies based on their primary patent classification. Figure 6 reveals that more than one-fifth of the 32,000+ citations to NIST come from patents related to Computer Hardware \& Software; and more than one-third are 
from that category plus Communications. Other major citing categories are Measuring \& Testing, and Semiconductors \& Electronics.

It is also interesting to note the distribution of citations across different technologies and NIST document types. For example, Measuring \& Testing and Semiconductors \& Electronics patents cite NIST peer reviewed papers extensively. The same is true for Biotechnology, Ceramics, and Chemicals patents. Meanwhile, Computer Hardware \& Software and Communications patents build much more extensively on NIST grey literature.

Figure 7 contains the same data as Figure 6, but divides the citations to NIST grey literature into the eight sub-categories outlined in the methodology section of the report. This figure reveals that a large number of citations from Computer Hardware \& Software and Communications patents are to Category 5 (Software/Standard Reference Databases/Algorithms). Many of these citations are to FIPS documents such as the Secure Hash Standard (FIPS 180) and the Advanced Encryption Standard (FIPS 197). Another highly cited category of NIST grey literature is Category 7 (Official NIST Publications). Many of these citations are to NIST documents such as NISTIRs and NIST special publications, which often describe the contents of the FIPS documents from Category 5.

Figures 8 and 9 show the distribution of citations to NIST by citing assignee. Figure $8-$ which is divided into the four main NIST document types - reveals that IBM and Microsoft cite NIST most extensively, followed by Calcitec (a medical devices firm), 3M and Micron Technology. This figure also reveals that the different assignees cite different types of NIST outputs. Both IBM and Microsoft build extensively on NIST grey literature, as do Intertrust, Intel, Cisco and Hewlett-Packard. Meanwhile, Calcitec builds primarily on NIST peer reviewed papers, as do the University of California and Michigan State University, plus the US Department of Commerce (which includes citations from NIST's own patents to NIST papers). NIST's patents are cited most heavily by 3M, Western Digital and Cree.

Figure 9 is the same as Figure 8, but with citations to NIST grey literature divided into the eight subcategories. This figure highlights the extensive influence of the Software/Standard Reference Databases/Algorithms subcategory (Category 5) on the patents of IBM and Microsoft, plus the patents of First Data, Cisco and Oracle. It also highlights the influence of NIST Educational Networking (Category 4), such as workshops and conferences, on the patents of Microsoft, Intertrust and Assa Abloy.

Figures 10 and 11 show the distribution of citations to NIST according to the industry of the citing assignees. Note that these industries are not the same as the technologies examined earlier in Figures 6 and 7. For example, Microsoft is categorized as being in the 
Software industry, based on its primary industry classification. Hence, Microsoft's patents are all considered as part of the Software industry in Figures 10 and 11. However, while Microsoft is a software company, it has patents across a wide range of technologies (as defined by patent classifications), which were used in the generation of Figures 6 and 7 .

Figure 10 shows that Universities cite NIST technical outputs most extensively, followed by Semiconductor companies, Software companies, and Communications companies. Other industries that cite NIST outputs extensively are Medical Equipment/Instruments, Computer Systems and Electronics. Figure 10 also reveals that Universities cite primarily NIST peer reviewed papers, as do Medical Equipment/Instruments companies. Meanwhile, Semiconductor, Software and Communications companies build more extensively on NIST grey literature. Semiconductor companies also cite NIST patents extensively, as do Electronics and Computer Peripherals/Storage companies.

Figure 11 breaks out citations to NIST grey literature according to the various subcategories of this literature. This figure shows that Semiconductor, Software, Communications and Computer Systems companies have large numbers of citations to NIST Educational Networking (i.e. workshops and conferences). Companies in these industries also cite Official NIST Publications, and Other NIST Publications, extensively.

Overall, the organization-level results discussed above highlight the significant increase in citations from US patents to NIST technical outputs. These citations are from patents owned by organizations across a broad range of industries, describing a wide variety of technologies. The results above also demonstrate how the increase in citations to NIST outstrips citations to comparable document sets. This suggests that NIST's technical outputs have had a strong, and growing, impact on technological developments.

\section{Document-Level Results}

The previous section of the report focuses on results at the level of the entire portfolio of NIST technical outputs. In this section, we report results at the level of individual NIST outputs. The objective of this section is to highlight individual NIST documents that have had a particularly strong impact on subsequent technological developments. These results are divided according to the eleven detailed categories of NIST outputs described in the methodology section of the report (i.e. NIST assigned patents; NIST government interest patents; NIST peer reviewed papers; plus eight categories of NIST grey literature).

Table 1 lists the NIST assigned patents with the highest Citation Index values. As outlined earlier in the report, the Citation Index is a normalized impact indicator. It measures how many times patents have been cited as prior art by subsequent patents, compared to peer 
patents from the same year and technology. For example, the patent at the head of Table 1 (US \#5,356,756) has been cited by 110 subsequent patents. The mean number of citations for all 1994 patents in the same patent classification is 14.6 . Hence, the ' 756 patent has a Citation Index of 7.53 (110/14.6), showing it has been cited 7.53 times as frequently as expected. This patent describes materials processing for thin films, designed for use in sensors, electronic devices and sample analysis.

The second and third patents in Table 1 (US \#5,795,782 and US \#6,015,714) are part of the same patent family (i.e. they are related to the same underlying invention). They describe a method for rapid DNA analysis, and have both been cited by more than 100 subsequent patents, five times as many citations as expected given their age and technology. Looking further down the list of patents in Table 1, the one with the highest absolute number of subsequent citations is US \#6,088,679. This patent describes a business process system for workflow management. It has been cited by 233 subsequent patents, but this is only twice as many expected, since it is in a rapidly expanding area of patenting (i.e. business methods), in which citation rates are very high.

Table 2 shows the NIST government interest patents that have the highest Citation Index values. There are a number of patents in this table with very high Citation Indexes, notably the patent at the head of the table (US \#7,330,404). This patent has been cited by 127 subsequent patents since it was issued in 2008, which is more than 40 times as many citations as expected given its age and technology. It describes a transducer for use in optical storage (for example in disk drives), and is assigned to Seagate Technology - one of a number of highly cited Seagate patents in Table 2. Other companies with a series of highly cited NIST government interest patents include ConforMIS (whose patents describe treatment of cartilage in joints); Bind Biosciences (controlled drug delivery); and General Electric (phosphor blends for LED devices).

NIST-sponsored peer reviewed papers that have been cited frequently in US patents are shown in Table 3. The paper at the head of this table has been cited by 277 subsequent patents (and over 5,000 papers, according to Google Scholar). It was published in Science in 1995, and describes sequencing of the H.influenzae genome (the first example of a freeliving organism having its entire genome sequenced).

The second paper listed in Table 3 does not report original research, but is instead a literature survey of face recognition technology. It has been cited by 161 patents. There are three further papers in Table 3 that have been cited by more than 100 patents. They are a 1996 paper describing a membrane filter for use in DNA analysis (137 patent citations), and two papers from the same NIST research group describing methods for measuring nanoscale distances (120 and 116 patent citations). The most highly cited recent NIST peer 
reviewed paper is the 'NIST Definition of Cloud Computing'. This was published in the Communications of the ACM in 2010, and has already been cited by 68 subsequent US patents.

Tables 4-11 list the individual items of NIST grey literature that have been cited most frequently by US patents, with each table focusing on a different category of this literature. Table 4 contains the most highly cited NIST Educational Networking publications (i.e. workshops, presentations, conference papers etc). The most highly cited of these publications is a 1996 presentation describing a proposed federal public key infrastructure (PKI) for secure communications. It has been cited as prior art by 28 subsequent patents. It is one of a number of papers in Table 4 concerned with PKI technology. The table also contains highly cited papers describing frameworks for information infrastructures, equipment operator training, and detecting intrusive behavior in networks.

Table 5 lists the NIST Software/Databases/Algorithms publications cited most frequently by subsequent US patents. The most highly cited are the Federal Information Processing Standards (FIPS) publications outlining the Advanced Encryption Standard (FIPS 197); the Secure Hash Standard (FIPS 180); and the Digital Signature Standard (FIPS 186). These standards documents have been cited by hundreds of subsequent patents, and are the most highly cited individual items of NIST grey literature.

The NIST Standard Reference/Resource Materials cited most frequently in patents are shown in Table 6. The most highly cited is the NIST 'Dictionary of Algorithms and Data Structures', which has been cited by 67 subsequent patents. Also highly cited are the redblack tree (48 patent citations), and the Bloom filter (17 patent citations), which are respectively a specific data structure and an algorithm listed in this NIST dictionary.

Table 7 lists the Official NIST Publications (NISTIRs, Special Publications etc) that have been cited most frequently by US patents. The publication at the top of this list is a 1980 NBS technical note describing a Schottky diode bridge sampling gate, designed for measuring low level signals in circuits. This publication has been cited by 101 subsequent US patents. The second publication in Table 7 is 'The NIST Definition of Cloud Computing' with 67 citations from US patents. This is NIST's own publication of this definition, which also appeared in the Communications of the ACM (see Table 3). Other highly cited NIST Special Publications are concerned with block ciphers and public key infrastructure, both of which are related to cryptography.

The publications in Table 8 are NIST Joint Partnership Publications, meaning they were developed in collaboration with other organizations and agencies. The publication at the head of this table is entitled 'Federal Criteria for Information Technology Security', and 
dates back to 1992. It has been cited by 31 US patents since its publication. More recent is the second publication in Table 8 , which is a review of ultra-wideband technology published by NIST in partnership with DARPA in 2003. This publication has been cited by 24 subsequent US patents. In general, the publications in Table 8 are relatively old, some dating as far back as 1960.

The publications in Table 9 (Other NIST Publications) are generally more recent, with most being published post-2000. The most highly cited of these publications is from 2003, and has been cited by 66 US patents. It is entitled 'Intrinsic velocity and its relation to intrinsic conductivity'. Other highly cited publications include papers on biometrics (38 patent citations), thin films (34 patent citations), and internet security (30 patent citations).

Table 10 contains items of correspondence with NIST that have been cited by numerous subsequent US patents. The citation counts in this table are mostly quite low, with only four items being cited by more than 10 patents. Two of these four items are comments to NIST regarding modes of operation of the Advanced Encryption Standard (AES). They have been cited by 17 and 11 patents respectively. The other two items are a submission to NIST regarding the Galois/Counter Mode (GCM), used in cryptography; and a private communication to NIST dating back to 1978.

The citation counts in Table 11 (Publications mentioning NIST) are also relatively low, with the most highly cited single publication having been cited by 18 subsequent US patents. This is a paper published in 1999 that refers to the NBS phosphor standard samples. Table 11 also contains a discussion of the NIST Digital Signature Standard (15 patent citations); a proposed NIST standard for role-based access control (15 patent citations); and an analysis of the NBS data encryption standard (14 patent citations).

\section{Conclusions}

This report outlines the results of an analysis designed to evaluate the technological impact of technical outputs associated with NIST. The analysis is based on tracing forward from these NIST outputs to determine how frequently they have been cited by subsequent patents. This makes it possible to evaluate the extent to which NIST funded activities have helped form the foundation for a wide range of technologies.

The analysis presented in this report reveals that there has been a significant increase over time in citations from US patents to NIST technical outputs. These citations are from patents owned by organizations across a broad range of industries, describing a wide variety of technologies. Our analysis also demonstrates how the increase in citations to 
NIST outstrips citations to comparable document sets. This suggests that NIST's technical outputs have had a strong, and growing, impact on technological developments. 


\section{Appendix: Figures and Tables}

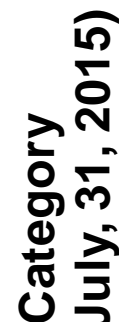

근든

o

동

它

응

은은

舟要

$\bar{z}$

잉

की

ฉ

응

$\div$

눙

$\pm$

స

西文

Ð $\frac{\mathrm{c}}{\mathrm{\sigma}}$

잉

证

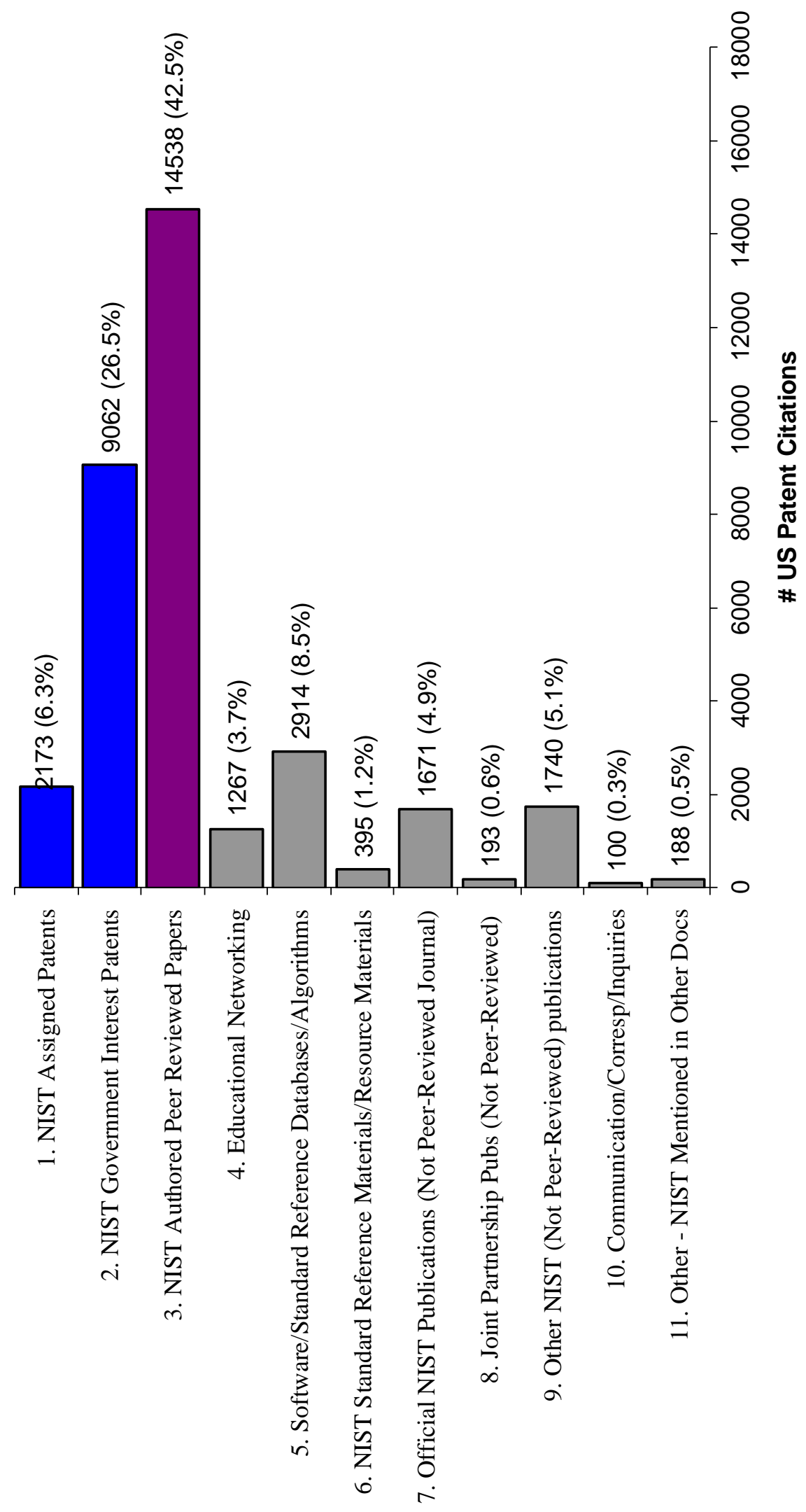



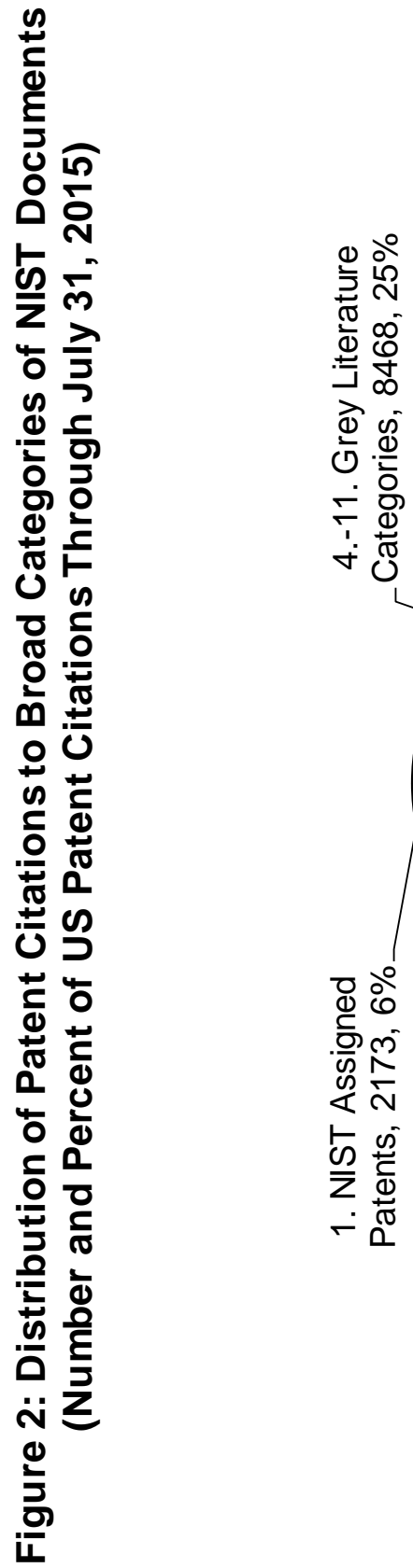

बे

(ए)

동

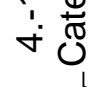

$\bar{\Phi} \stackrel{\infty}{m}$

过

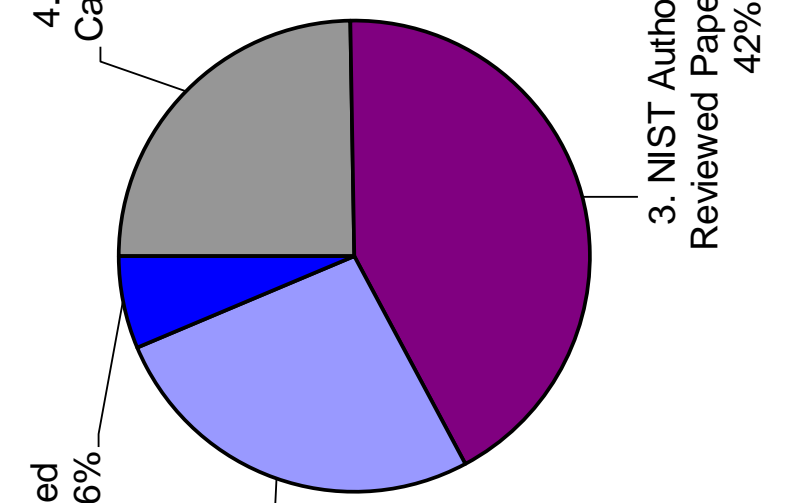

कू

安六

ळ

之

등

हू ه

홍

ర্

๘㐫

․ㅗ 


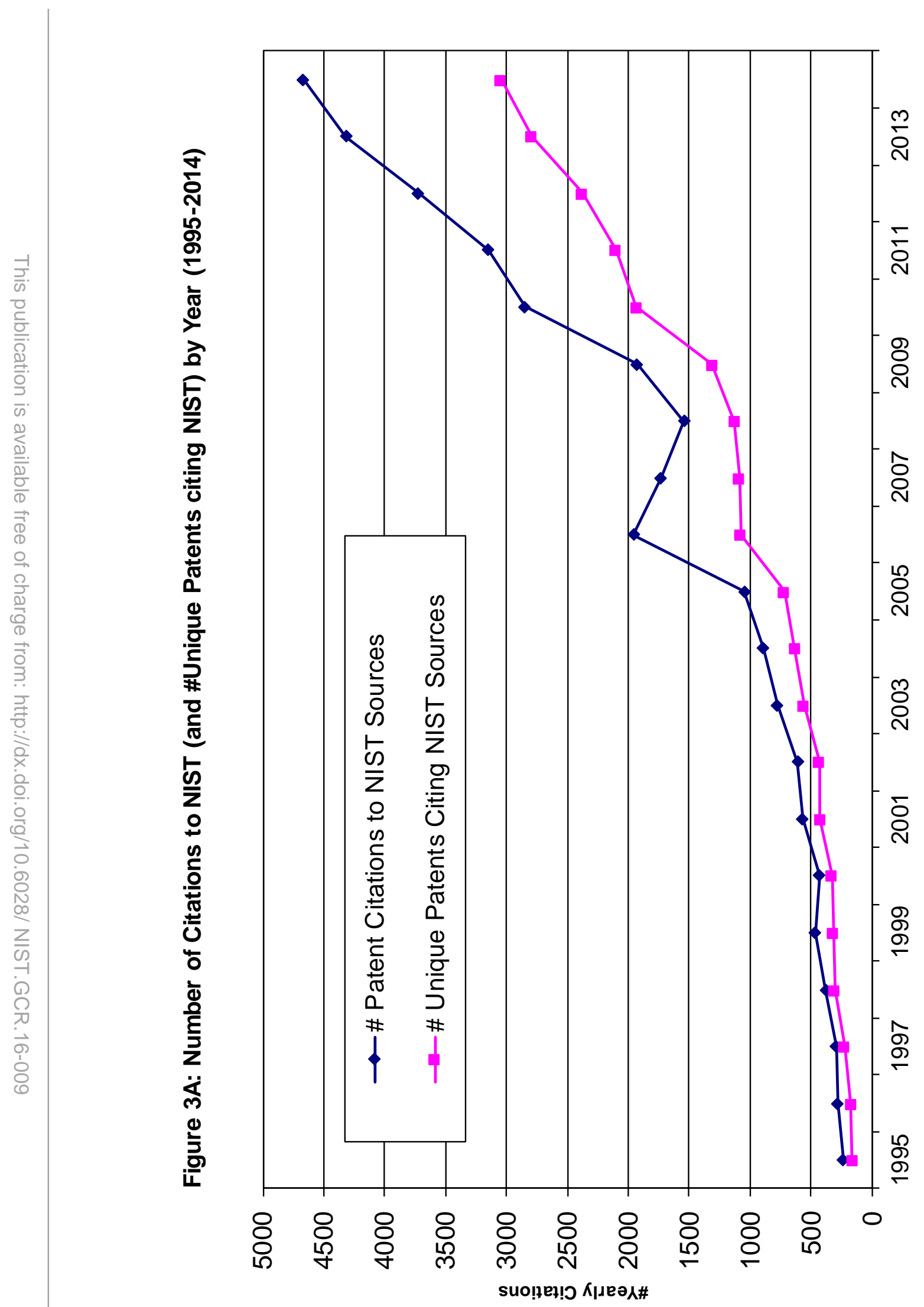




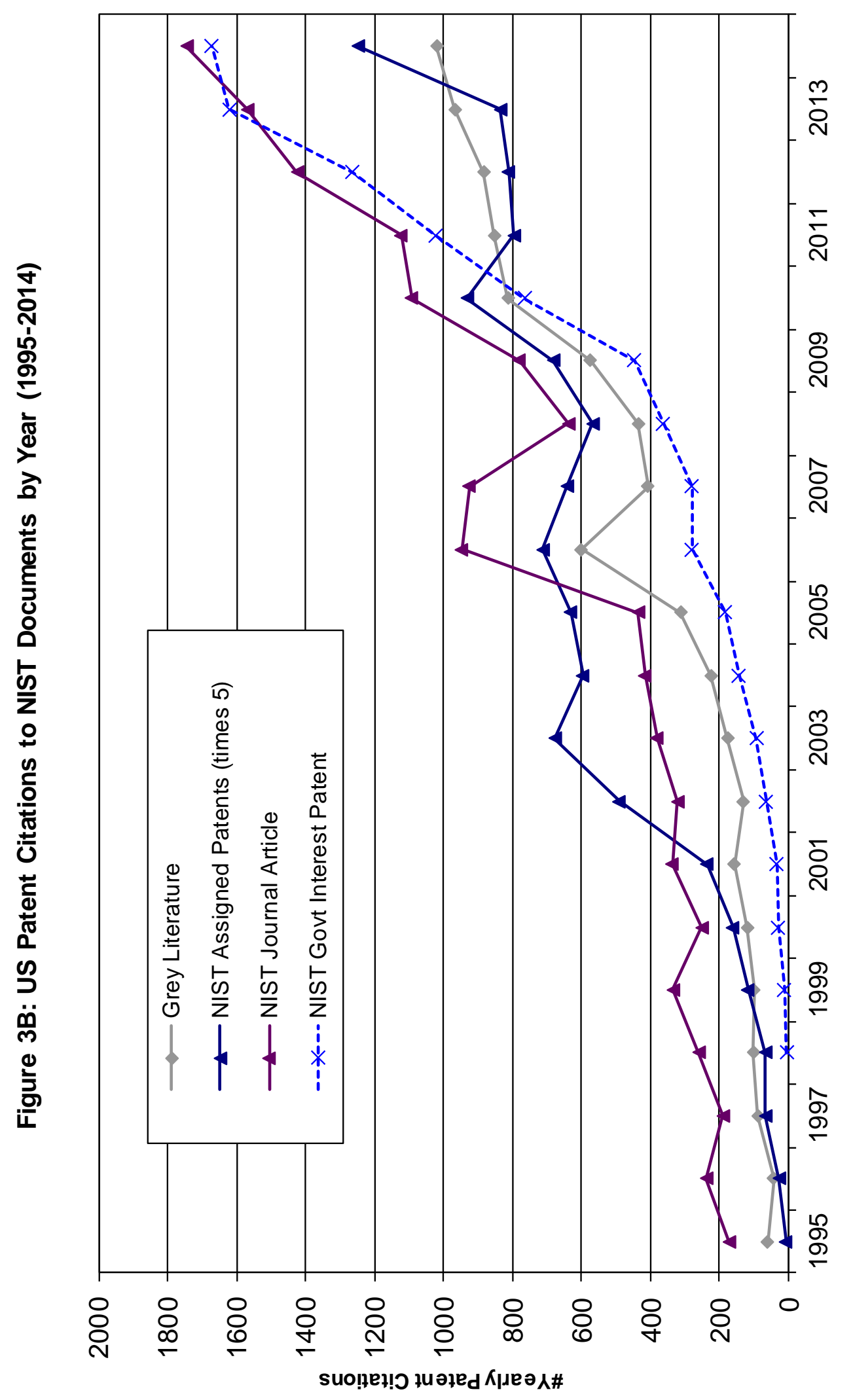




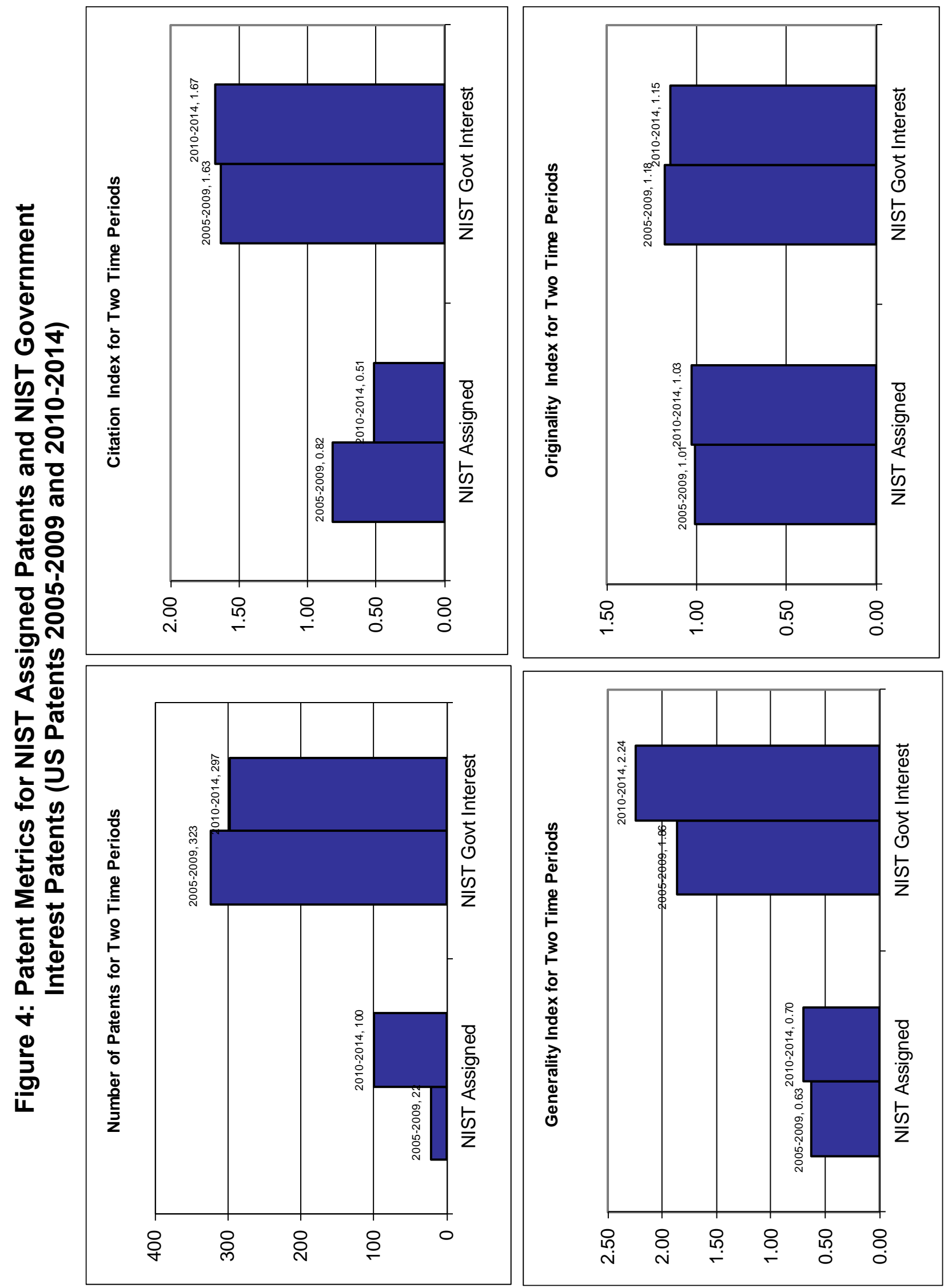




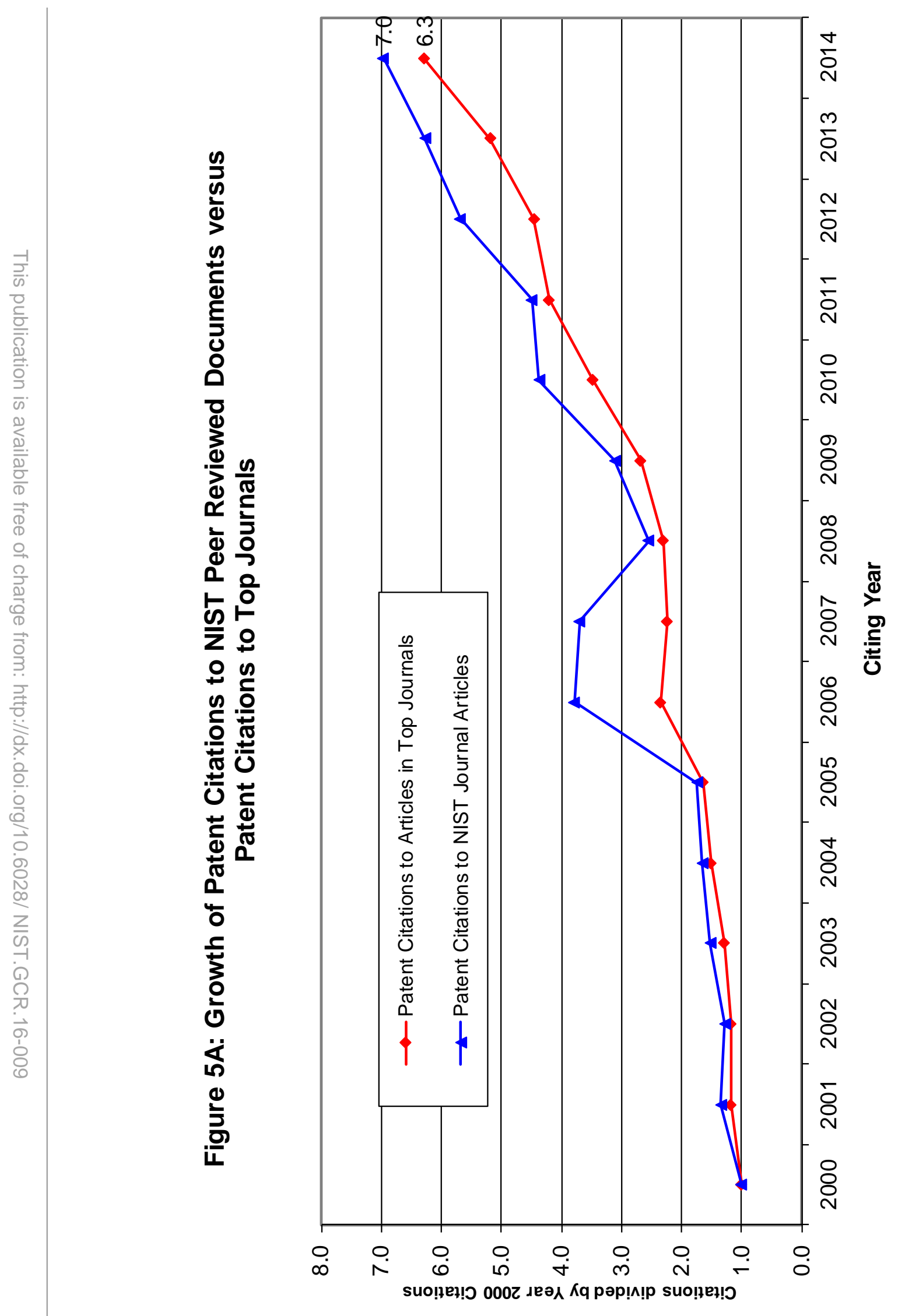




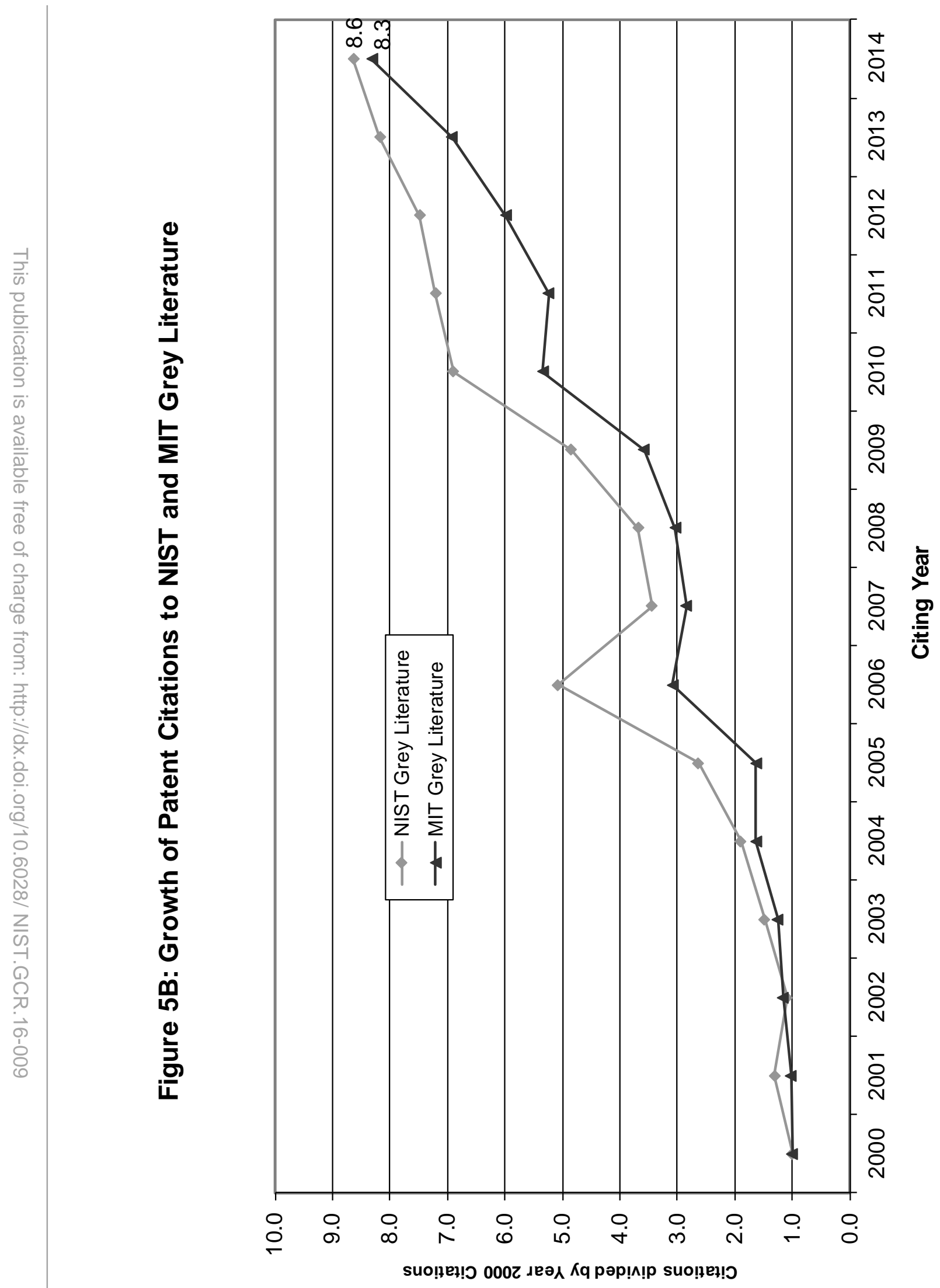




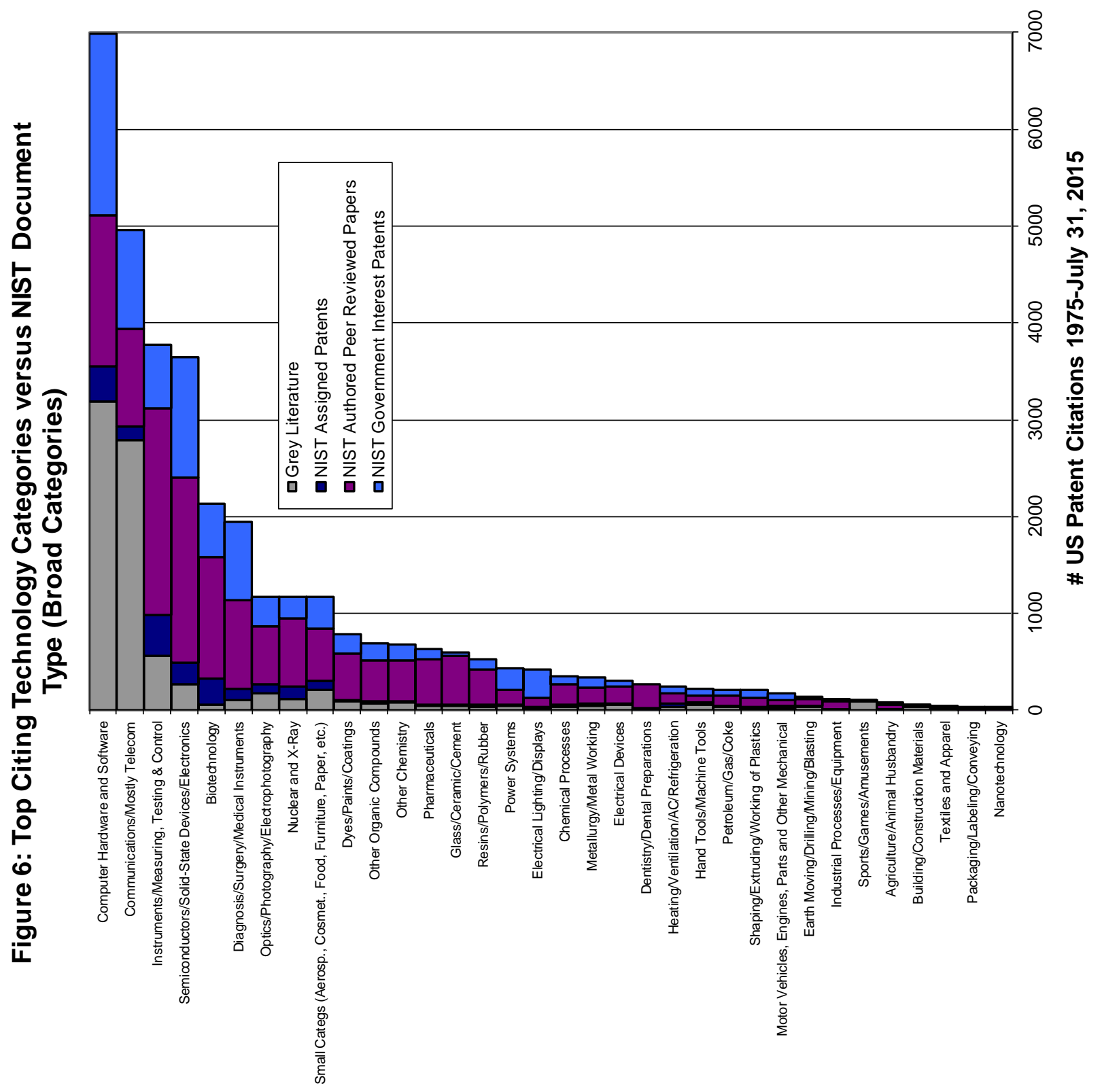




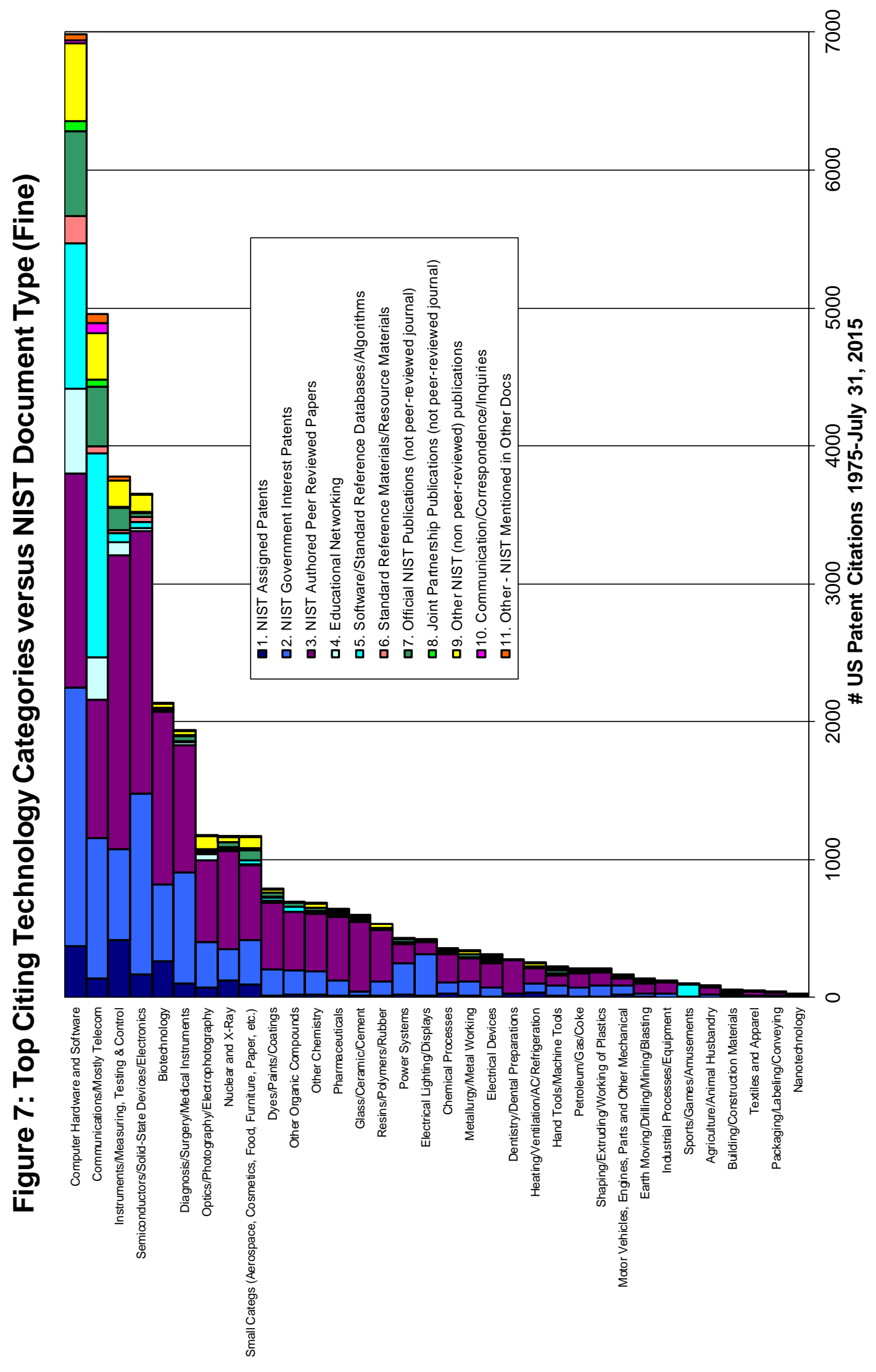




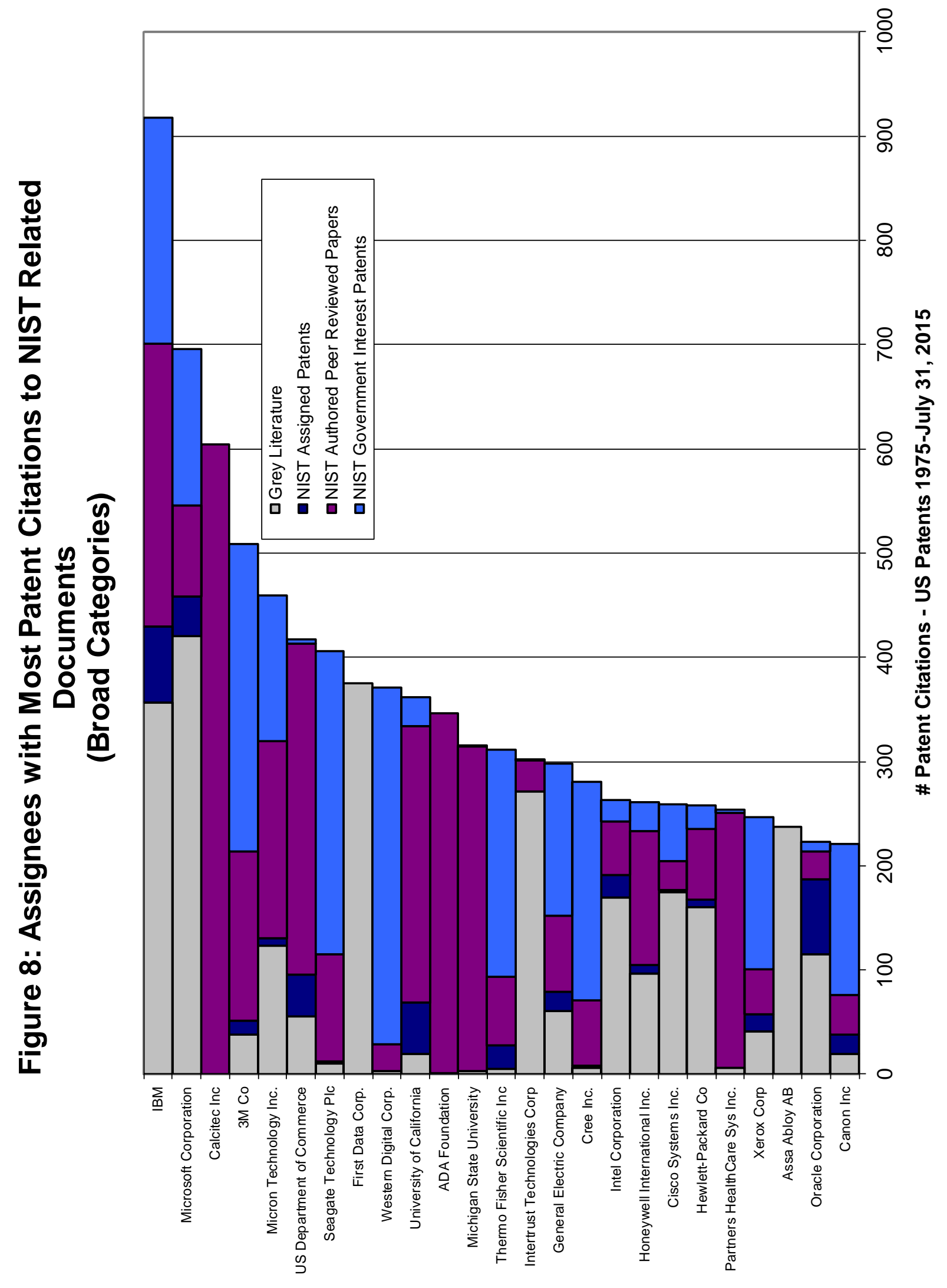




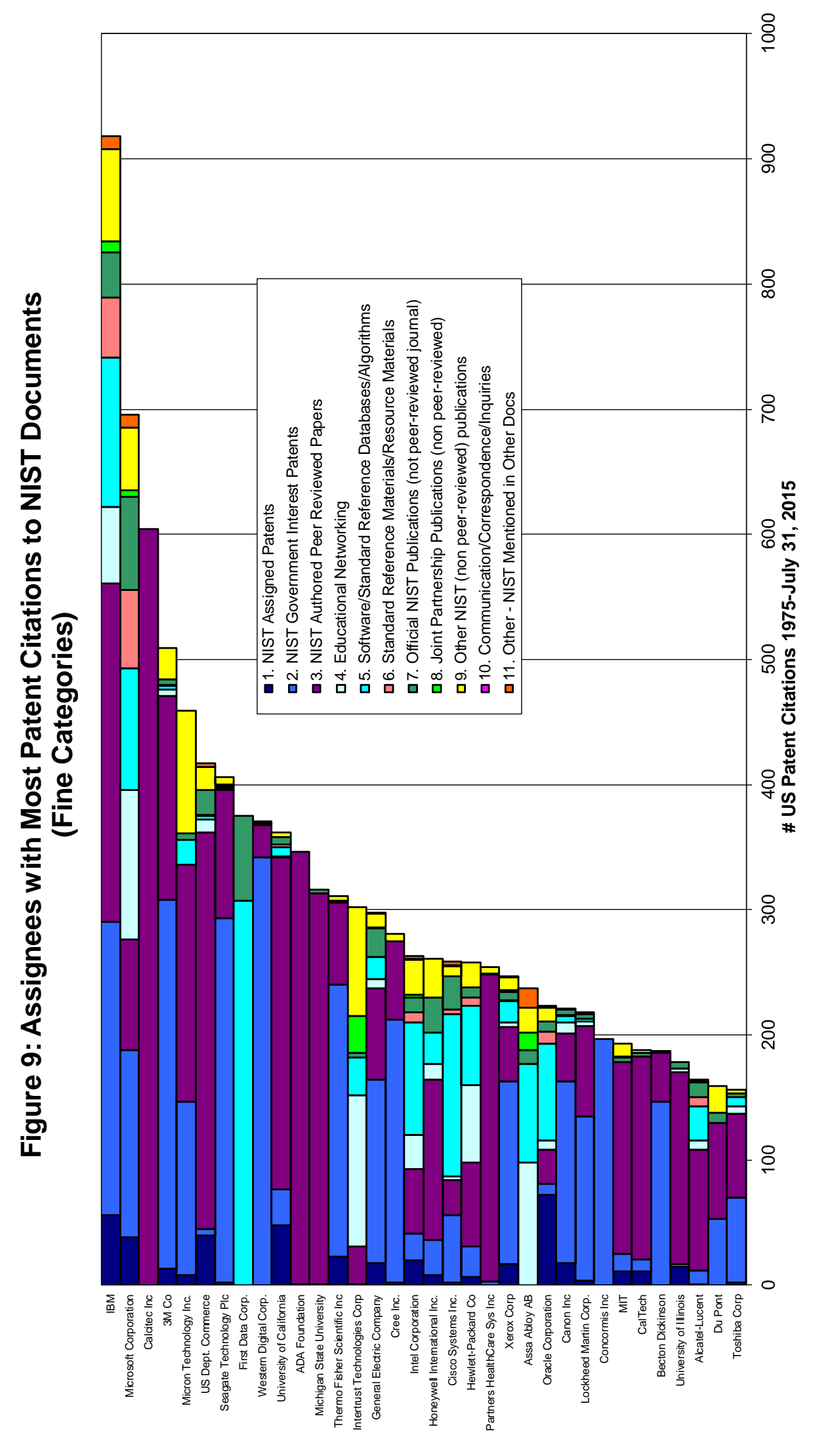




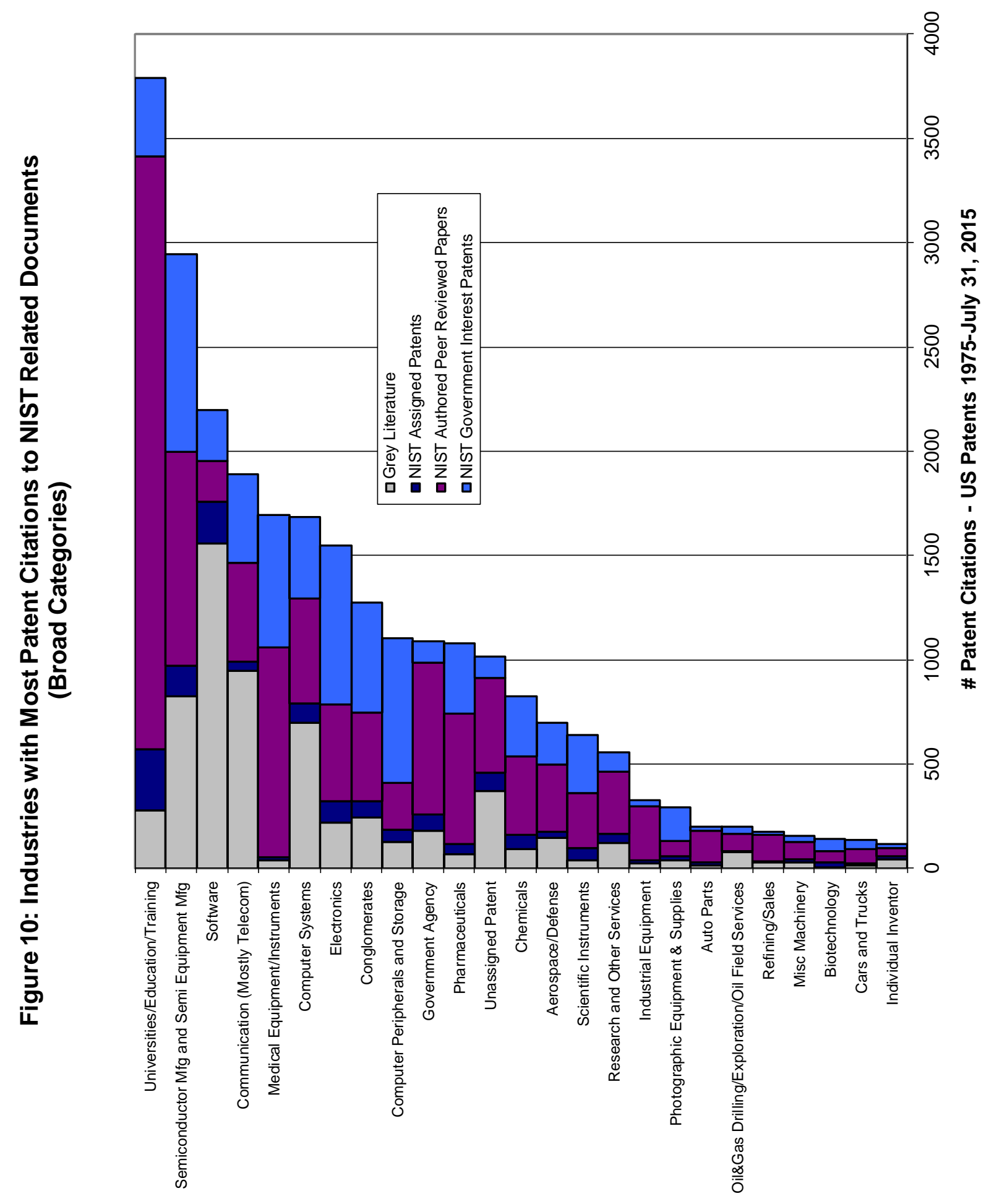




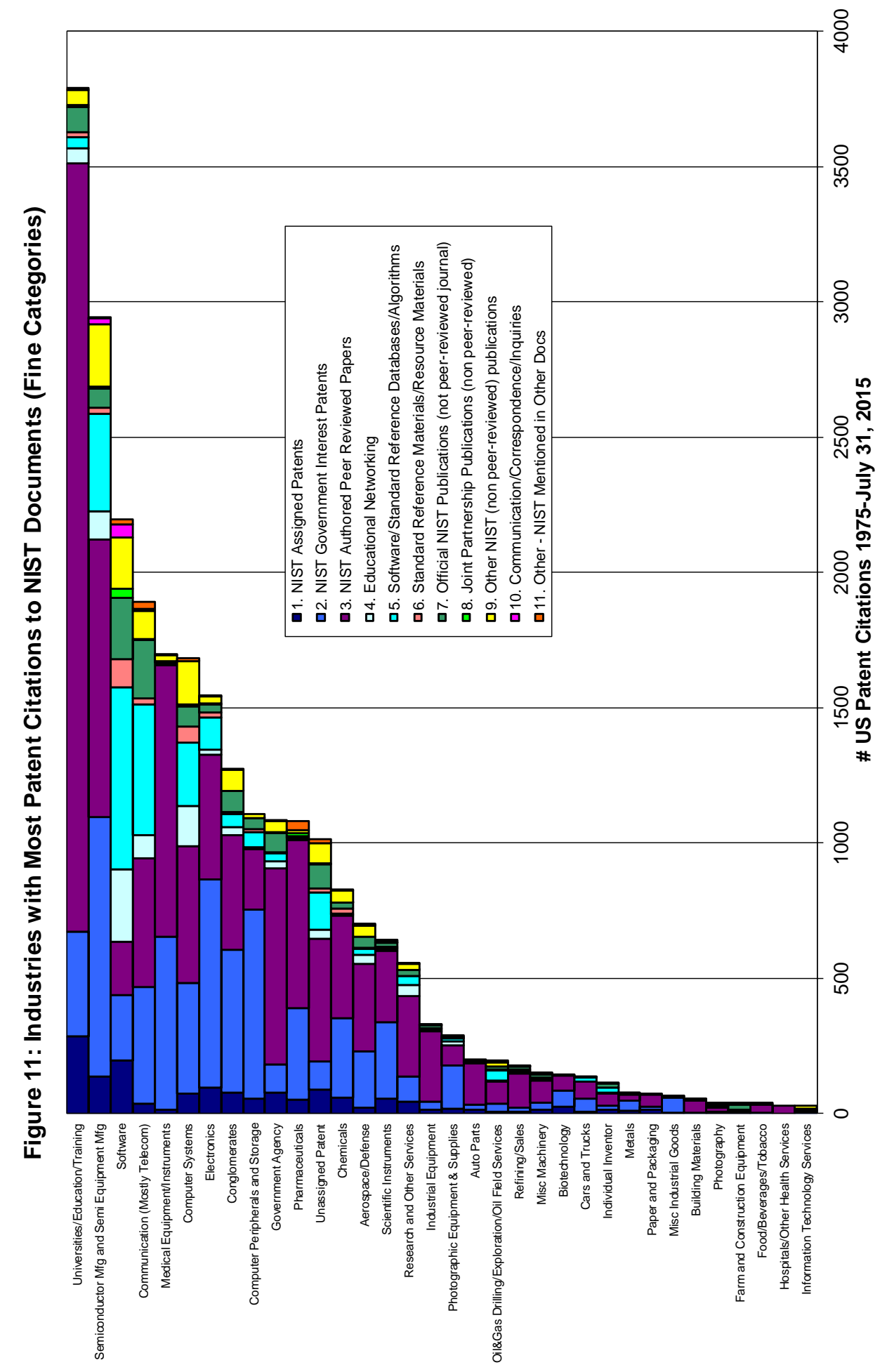




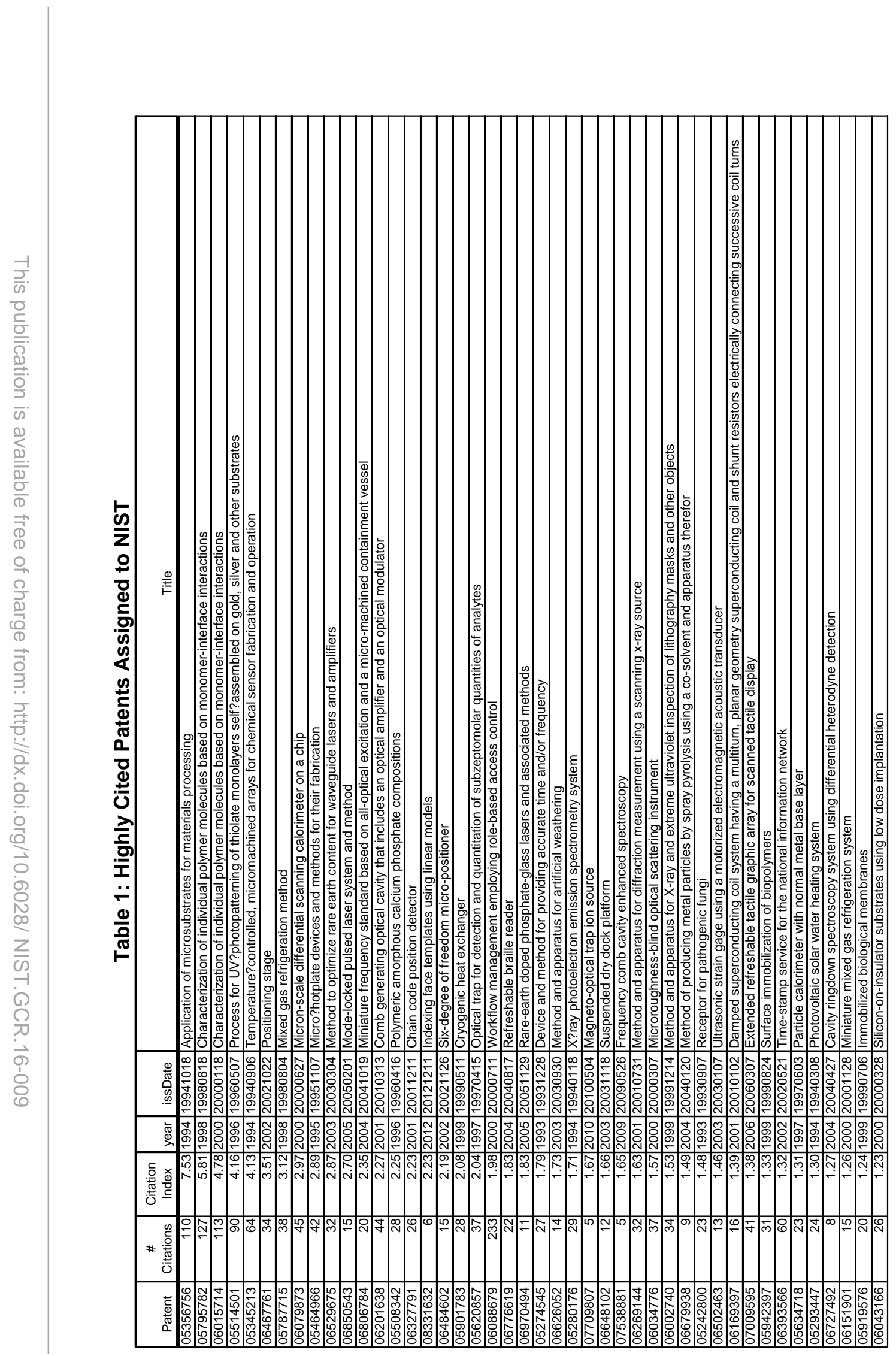




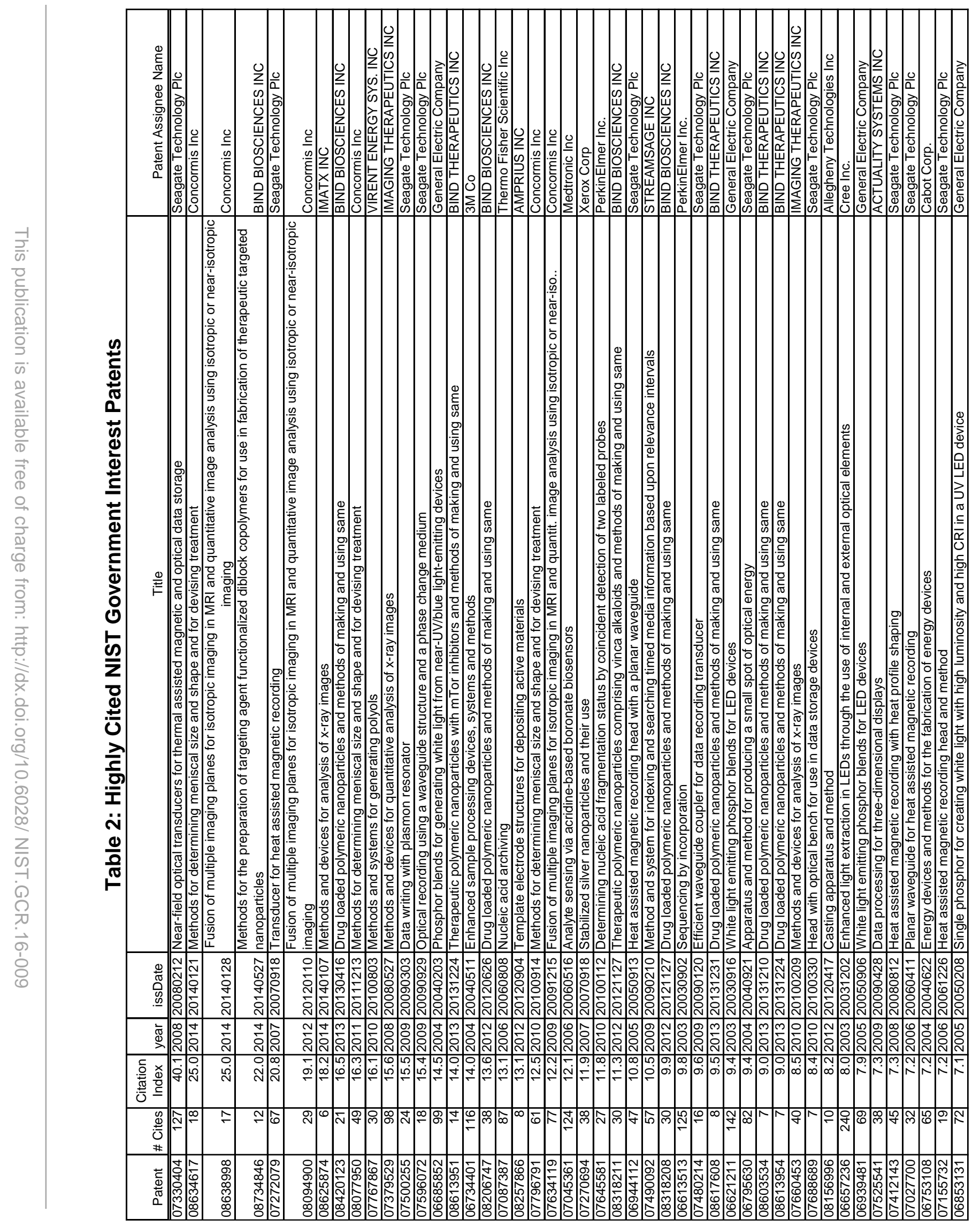




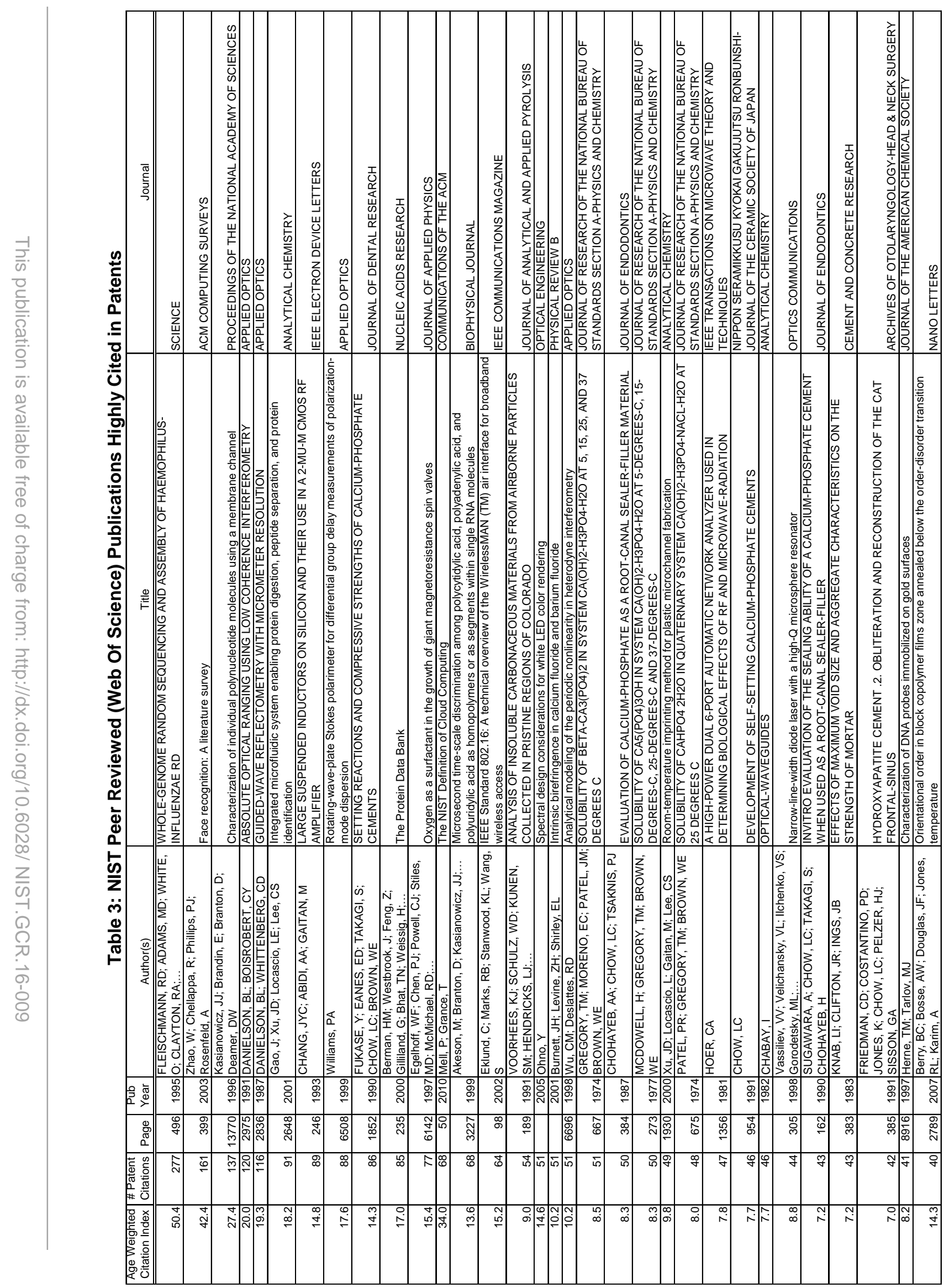




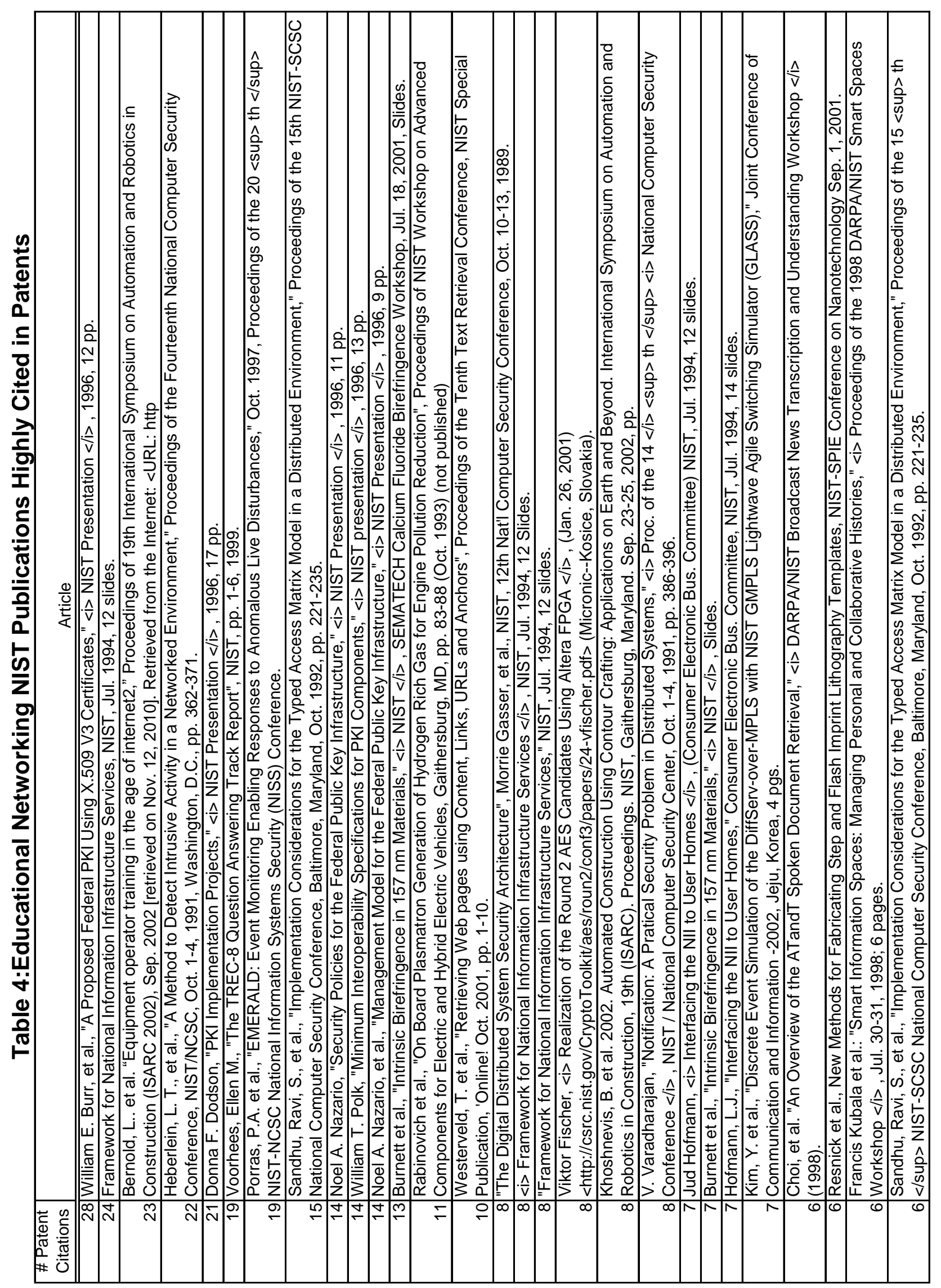




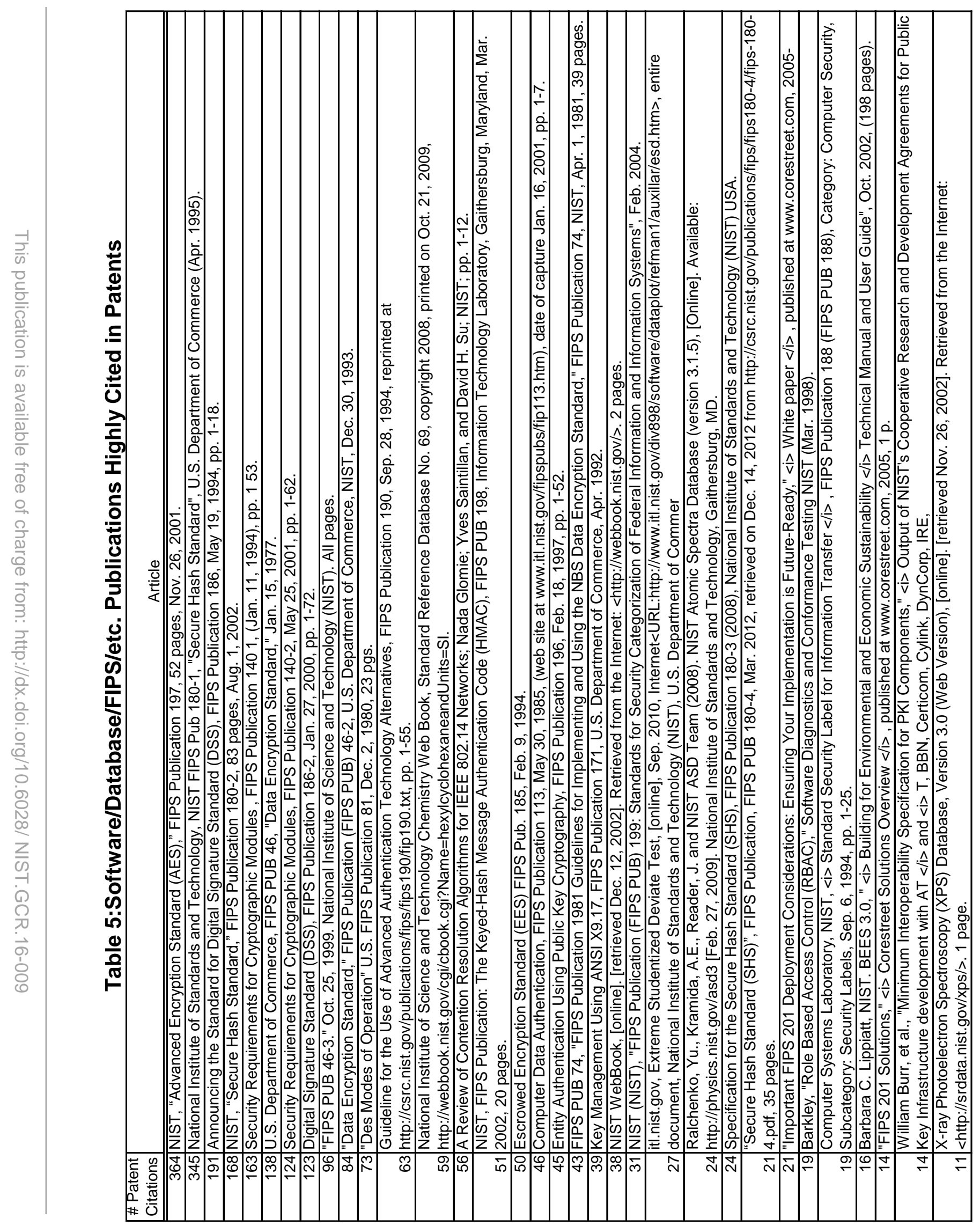




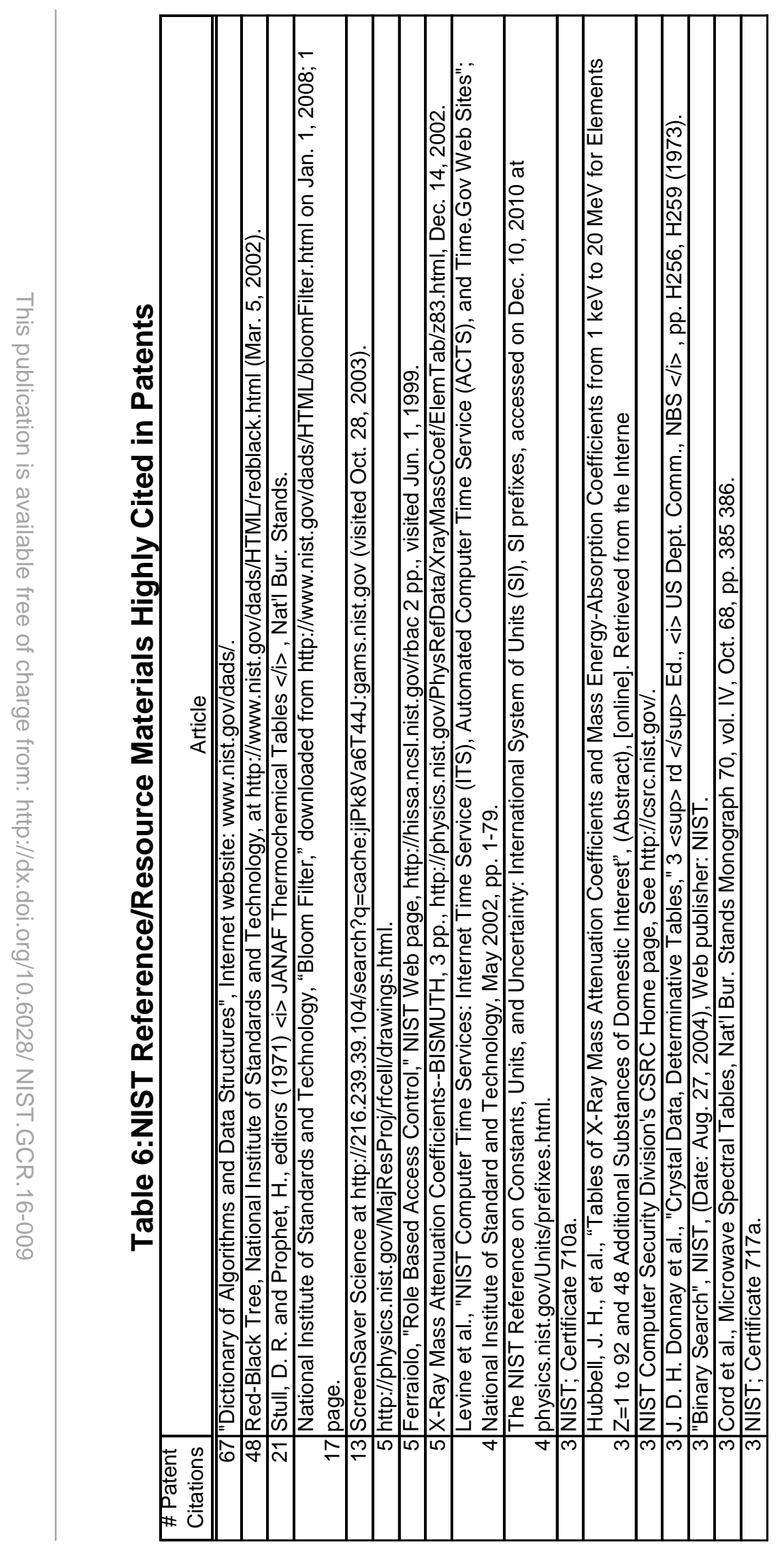




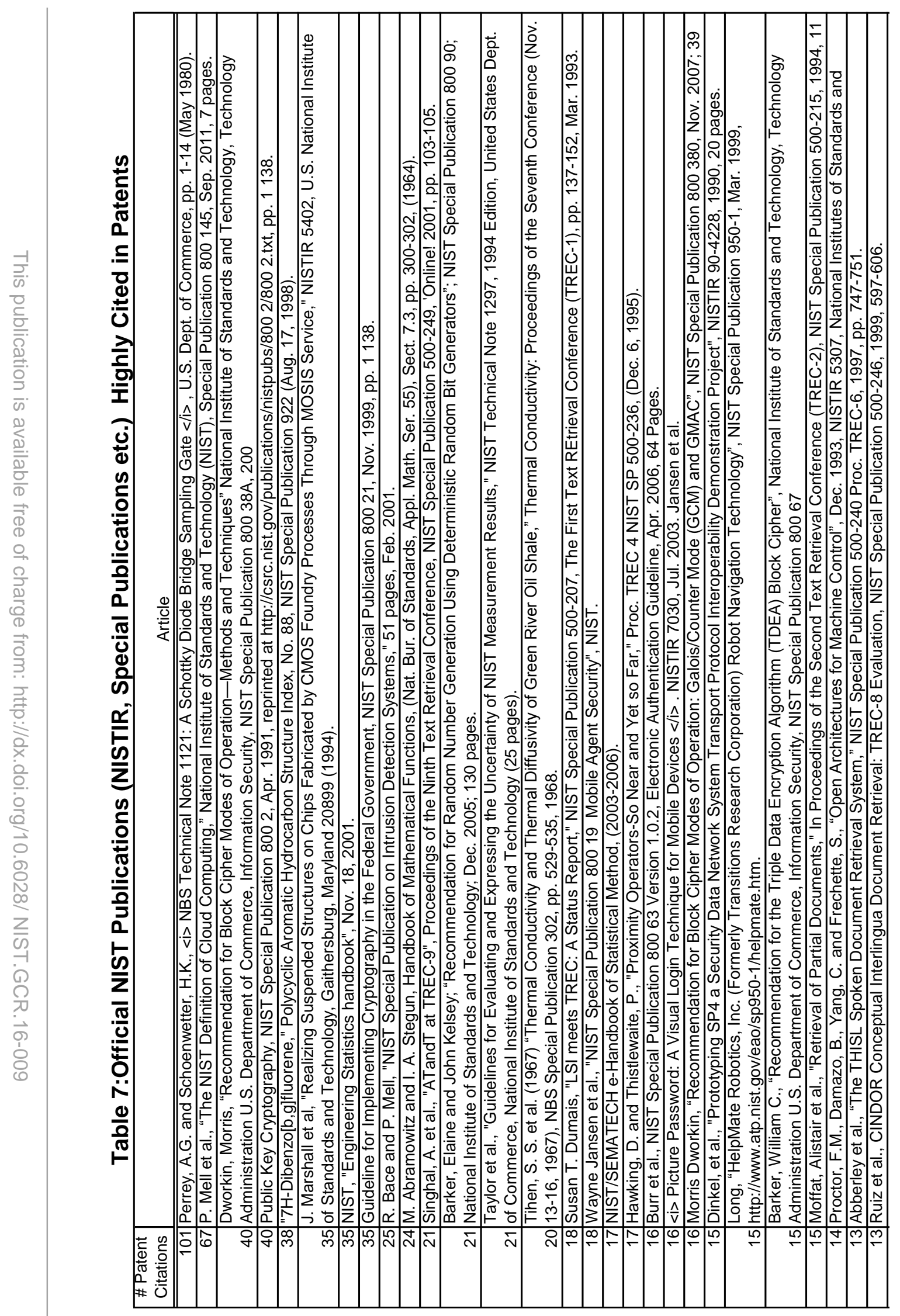




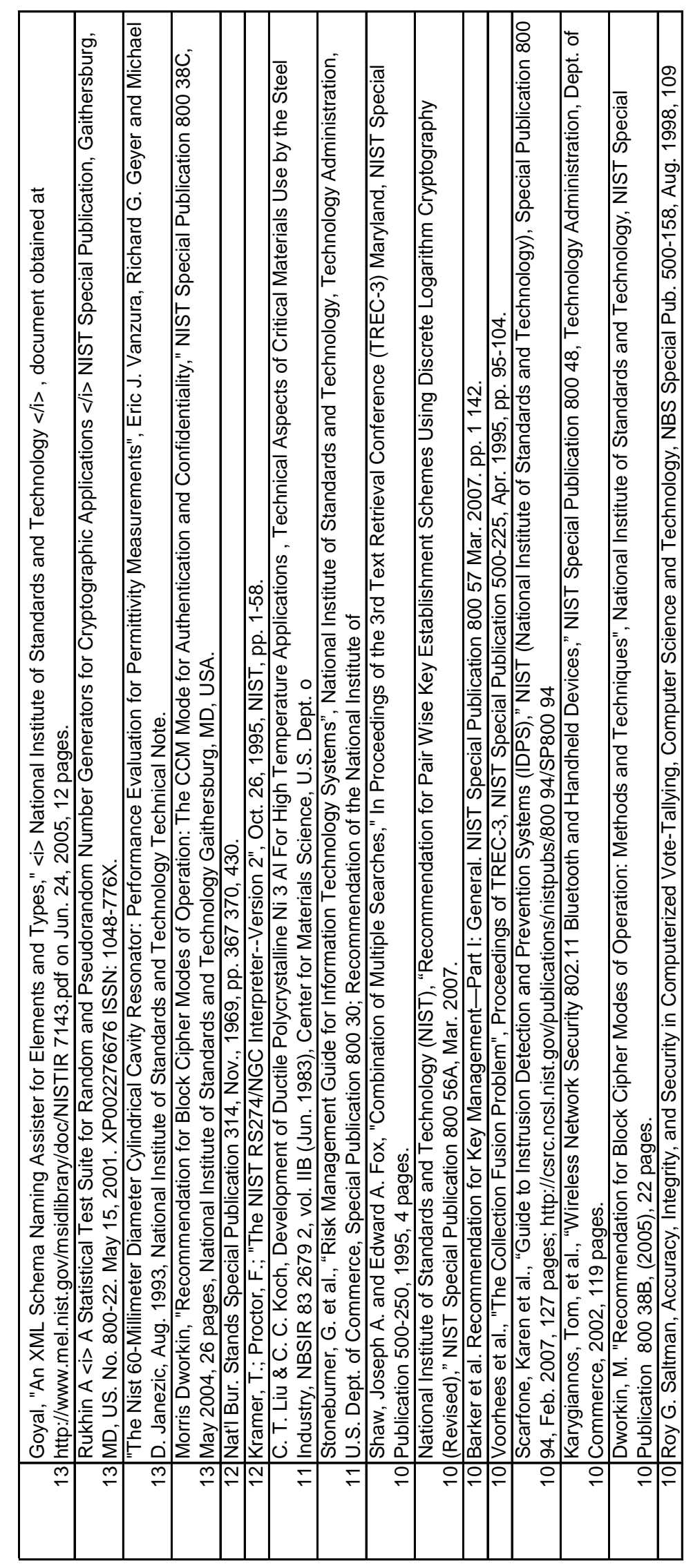




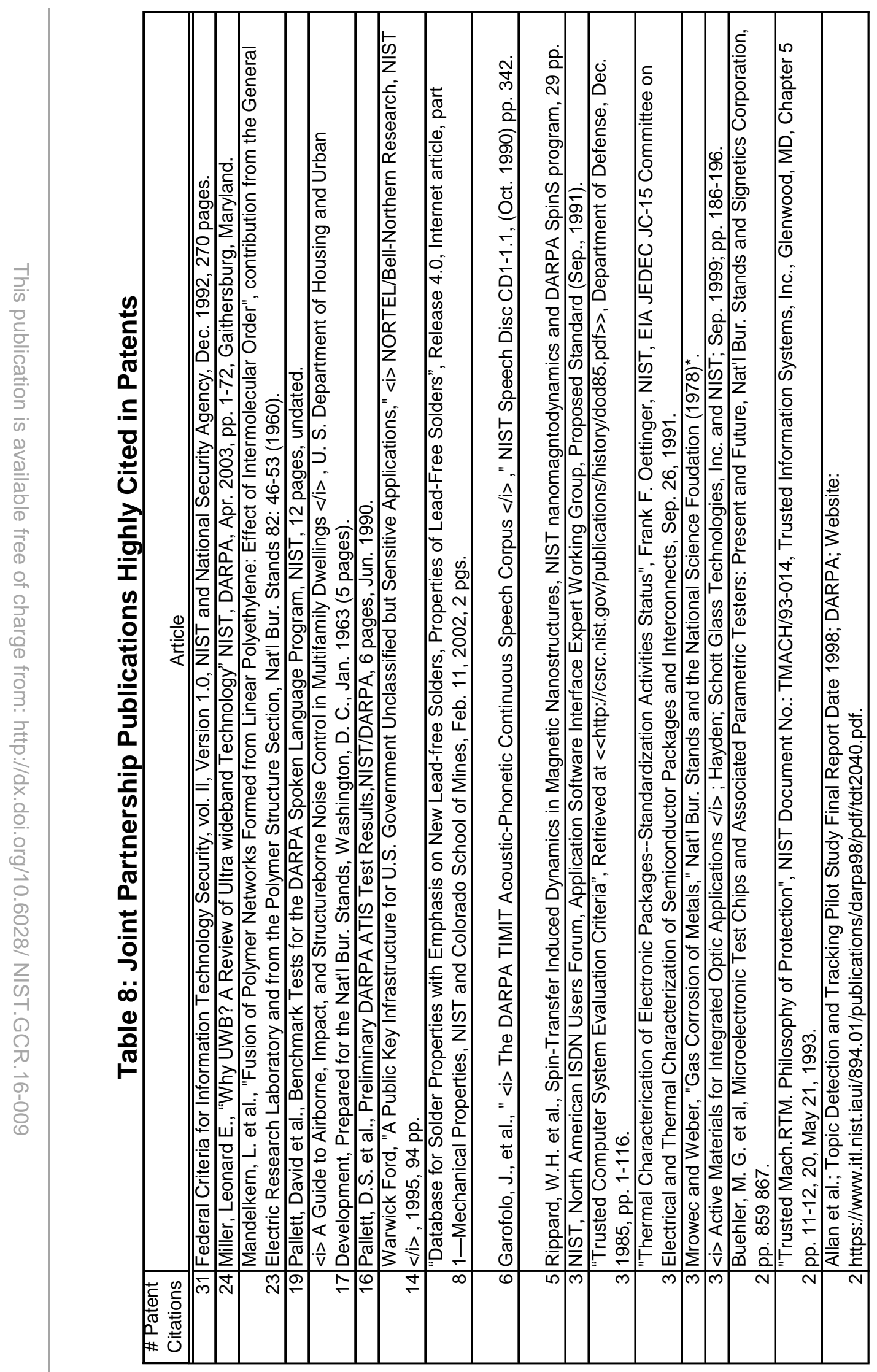




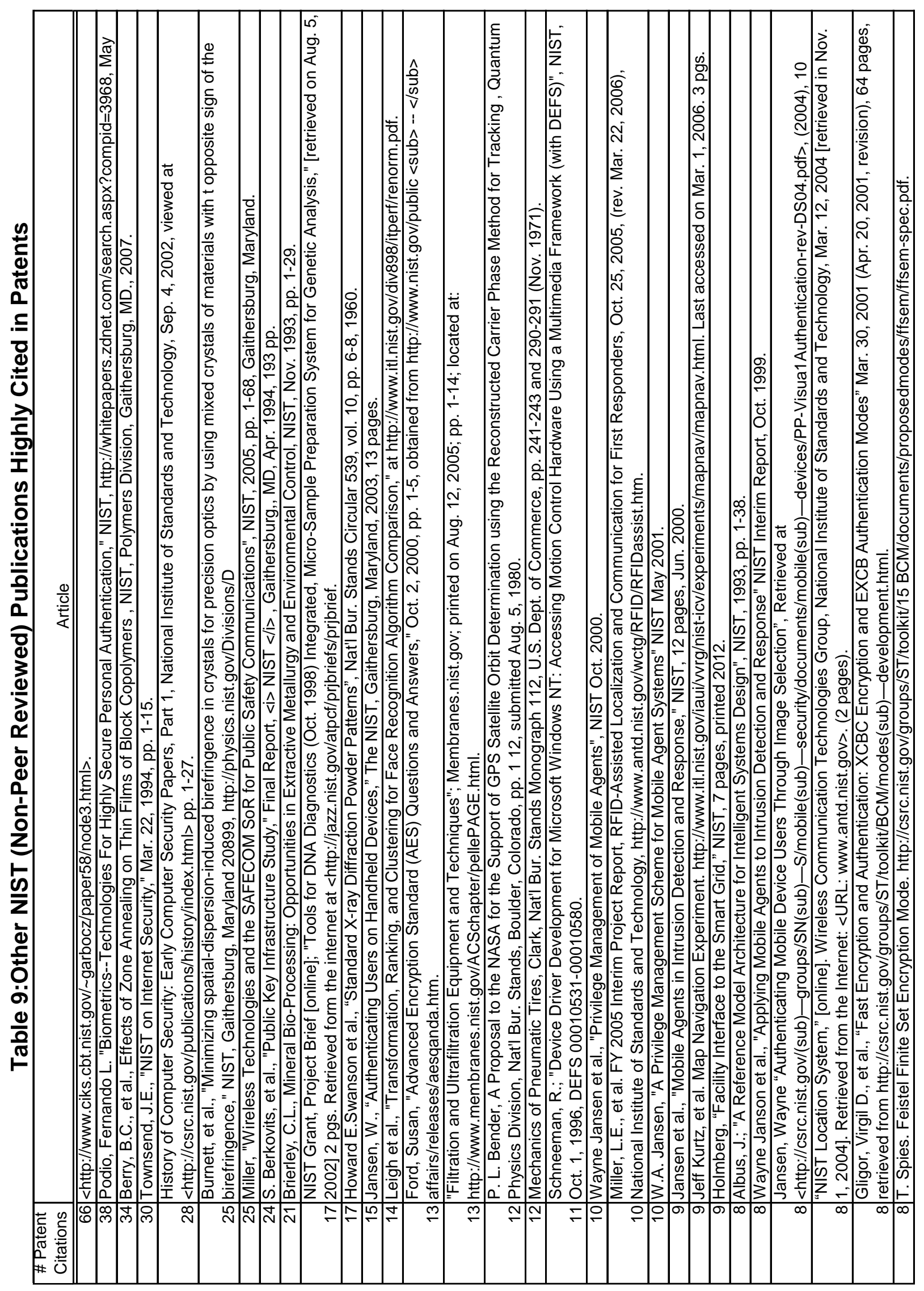




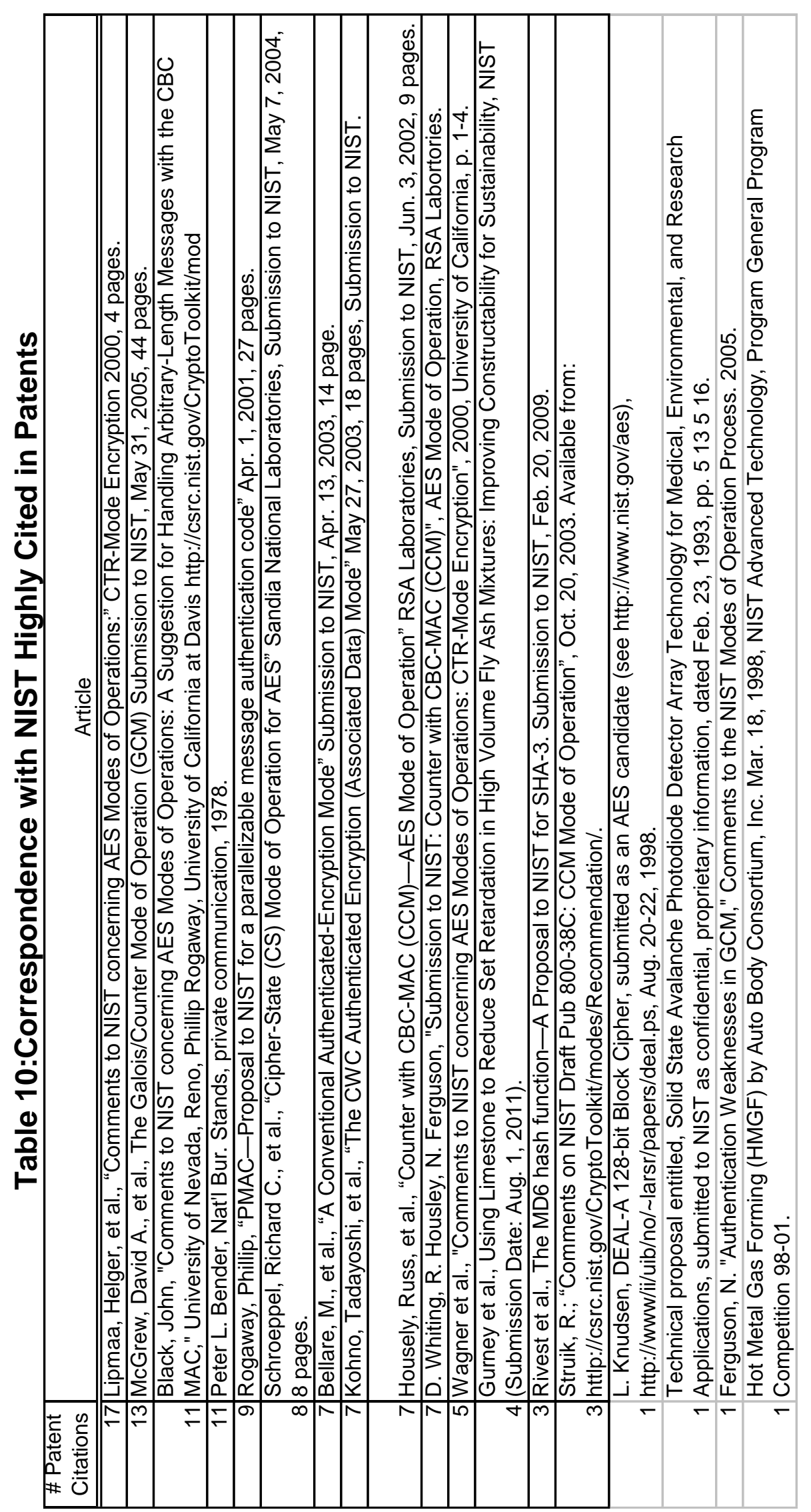




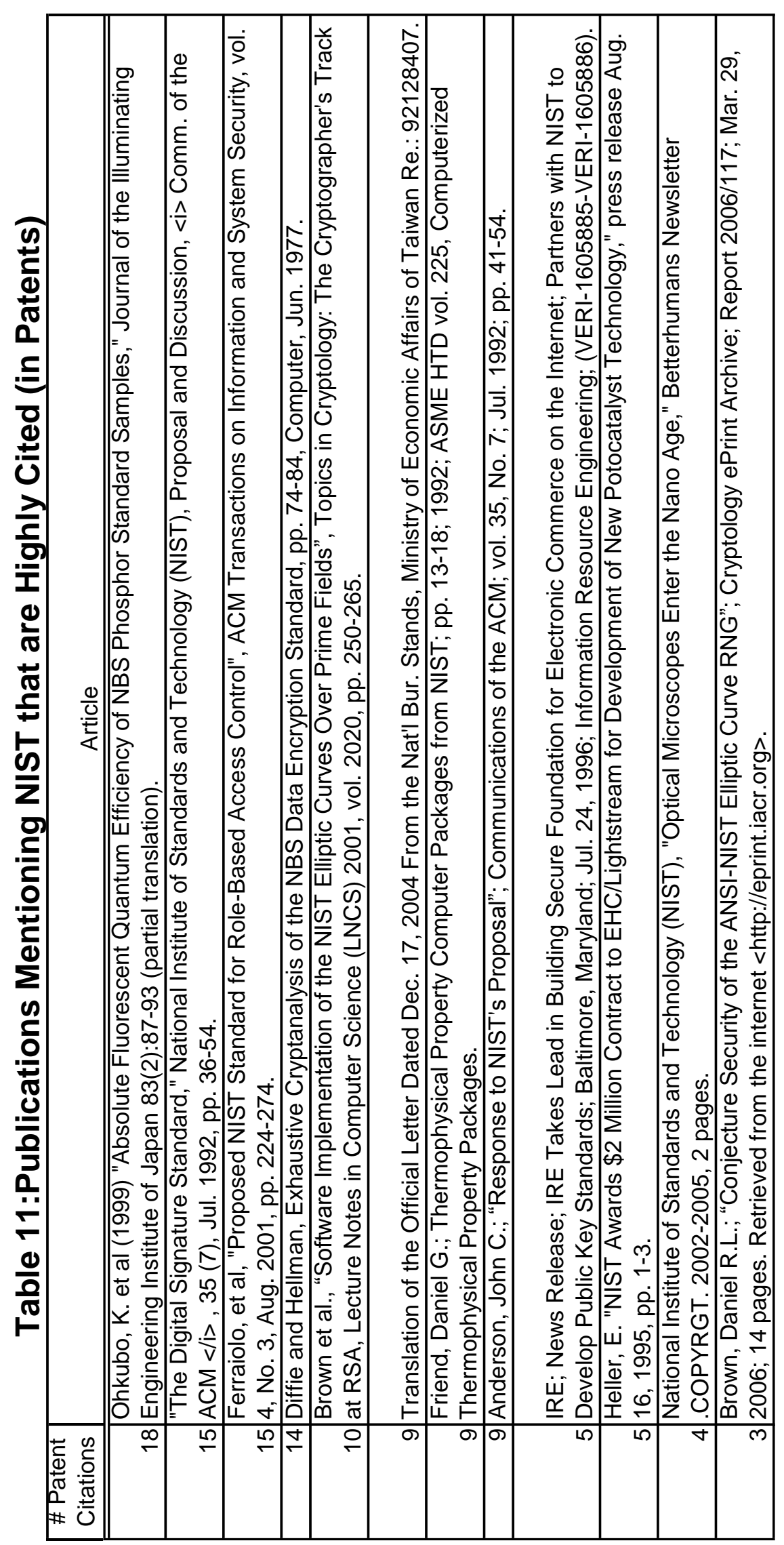

